OPEN ACCESS

Edited by:

Peter Dovc,

University of Ljubljana, Slovenia

Reviewed by:

Ute Knierim,

University of Kassel, Germany Johannes Baumgartner,

University of Veterinary Medicine

Vienna, Austria

*Correspondence:

Arthur Francisco Araújo Fernandes afernandes2@wisc.edu

Specialty section:

This article was submitted to

Livestock Genomics,

a section of the journal

Frontiers in Veterinary Science

Received: 28 April 2020

Accepted: 15 September 2020

Published: 21 October 2020

Citation:

Fernandes AFA, Dórea JRR and Rosa GJM (2020) Image Analysis and Computer Vision Applications in Animal Sciences: An Overview.

Front. Vet. Sci. 7:551269. doi: 10.3389/fvets.2020.551269

\section{Image Analysis and Computer Vision Applications in Animal Sciences: An Overview}

\author{
Arthur Francisco Araújo Fernandes ${ }^{1 *}$, João Ricardo Rebouças Dórea ${ }^{1}$ and \\ Guilherme Jordão de Magalhães Rosa ${ }^{1,2}$
}

'Department of Animal and Dairy Sciences, University of Wisconsin-Madison, Madison, WI, United States, ${ }^{2}$ Department of Biostatistics and Medical Informatics, University of Wisconsin-Madison, Madison, WI, United States

Computer Vision, Digital Image Processing, and Digital Image Analysis can be viewed as an amalgam of terms that very often are used to describe similar processes. Most of this confusion arises because these are interconnected fields that emerged with the development of digital image acquisition. Thus, there is a need to understand the connection between these fields, how a digital image is formed, and the differences regarding the many sensors available, each best suited for different applications. From the advent of the charge-coupled devices demarking the birth of digital imaging, the field has advanced quite fast. Sensors have evolved from grayscale to color with increasingly higher resolution and better performance. Also, many other sensors have appeared, such as infrared cameras, stereo imaging, time of flight sensors, satellite, and hyperspectral imaging. There are also images generated by other signals, such as sound (ultrasound scanners and sonars) and radiation (standard x-ray and computed tomography), which are widely used to produce medical images. In animal and veterinary sciences, these sensors have been used in many applications, mostly under experimental conditions and with just some applications yet developed on commercial farms. Such applications can range from the assessment of beef cuts composition to live animal identification, tracking, behavior monitoring, and measurement of phenotypes of interest, such as body weight, condition score, and gait. Computer vision systems (CVS) have the potential to be used in precision livestock farming and high-throughput phenotyping applications. We believe that the constant measurement of traits through CVS can reduce management costs and optimize decision-making in livestock operations, in addition to opening new possibilities in selective breeding. Applications of CSV are currently a growing research area and there are already commercial products available. However, there are still challenges that demand research for the successful development of autonomous solutions capable of delivering critical information. This review intends to present significant developments that have been made in CVS applications in animal and veterinary sciences and to highlight areas in which further research is still needed before full deployment of CVS in breeding programs and commercial farms.

Keywords: computer vision, sensors, imaging, phenotyping, automation, livestock, precision livestock, highthroughput phenotyping 


\section{INTRODUCTION}

Sighted animals, including humans, experience vision in a way that seems natural and automatic. Early in life, and quite often from the moment of birth, animals use their vision system to navigate the world around them, and to identify and interact with other animals, as well as their surrounding environment. Therefore, the vision system of an animal is constantly being trained and adapted so that it can be used for several tasks. For instance, in humans, this system works with the luminous signal being captured by the eye and transferred via the optic nerve to the brain, where it is processed and interpreted (1). This complex vision system can adapt to different light conditions autonomously while allowing us to focus on objects and to have a 3-dimensional representation of the world. But what would be vision for a computer and how can computer vision impact animal breeding and production? This review is divided into three sections. The first section provides a brief introduction to image analysis and computer vision, describing current developments and algorithms of interest. The second section describes common types of sensors available and their functionality. The third presents a historical view on applications in animal sciences, followed by examples and areas of current interest. The review closes with a discussion on areas that are currently of great importance for the improvement of computer vision system (CVS) applications in livestock improvement and production.

\section{OVERVIEW OF STRATEGIES TO WORK WITH DIGITAL IMAGES \\ What Is Digital Image Processing, Image Analysis, and Computer Vision?}

Digital Image Processing, Digital Image Analysis, and Computer Vision can be viewed as an amalgam of terms that very often are used to describe similar processes and applications, generating confusion regarding their meaning. Most of the confusion arises because these are interconnected fields that emerged with the development of technologies for digital image acquisition. For the sake of clarity, we can divide and define these three areas as follows.

\section{Digital Image Processing}

Digital Image Processing deals with capturing and translating a visual signal into a digital image. As such, it can be viewed as the area that studies the process of obtaining a visual signal of the world and transforming it in order to make it interpretable. It spans from the study of image formation, as a result of the acquisition of light signals by specifically designed sensors, to the interpretation of the image as an array of connected values. Therefore, digital image processing involves the conception, design, development, and enhancement of digital imaging algorithms and programs (2). As such, it is a discipline heavily based on physics and mathematics. The term can also be used to directly address the applications or techniques used for digital image manipulation, ranging from noise reduction, image equalization, image filtering, and other transformations used for preparing images for subsequent steps in an analysis pipeline or for enhancing images aesthetically. A group of techniques of great importance in digital image processing is edge and contour detection. Although there are several methods for edge detection, they all rely on the fact that edges are regions in an image where there is a drastic change in color/intensity along with a particular orientation $(2,3)$. These techniques are, in general, useful in many applications in image processing, such as image correction and sharpening (i.e., highlight of the edges) and in image analysis, such as identification of complex structures and matching of objects in an image with specific templates.

\section{Digital Image Analysis}

Digital image analysis, or just digital imaging, on the other hand, corresponds to the process of extracting meaningful information from an image (2). This information can be descriptive statistics from the image, ranging from global image metrics, such as color/brightness histograms and distribution, block statistics from regions/windows across the images, such as intensity, moments (mean, variance), and integral images, to the identification of more complex structures in the image. Such information extracted from the image analysis can be used then as input for imaging processing techniques, such as image sharpening (4), thresholding (5), smoothing and edge/contour enhancement (6). On the other hand, image processing techniques can also be applied prior to image analysis techniques. An example is the use of edge detection techniques in the process of identification of structures, such as lines and circles in an image (3). Another is for image segmentation, i.e., to divide an image into different regions, which can be simple image binarization (a division of the image into two regions, such as background and foreground) or multiple regions, such as different objects present in an image. There are several methods of image segmentation, but basically, they can be classified into methods that perform a global clustering of image pixels according to some criteria independent of spatial information (e.g., k-means clustering), and methods that account for more information, such as spatial, texture, color, edges, and shape, such as energy-based (graph-cuts) methods $(7,8)$.

\section{Computer Vision}

Computer Vision can be defined as the field that aims to describe the world through images by interpreting, reconstructing, and extracting properties from images, such as shapes, textures, densities, and distances (9). CVSs are also known as machine vision systems, visual image systems, or just image systems. Therefore, Computer Vision is essentially the development of artificial systems to handle visual problems of interest, and for such, it uses image processing and analysis techniques. Along with image analysis and processing, other areas such as Machine Learning and Pattern Recognition are also highly interconnected with Computer Vision.

Pattern Recognition is a field that studies not only images but also other signals, such as sound and texts. As the name suggests, it is an area dedicated to the study of patterns that may appear in any given signal. In the context of imaging, pattern recognition is generally studied within image analysis as 


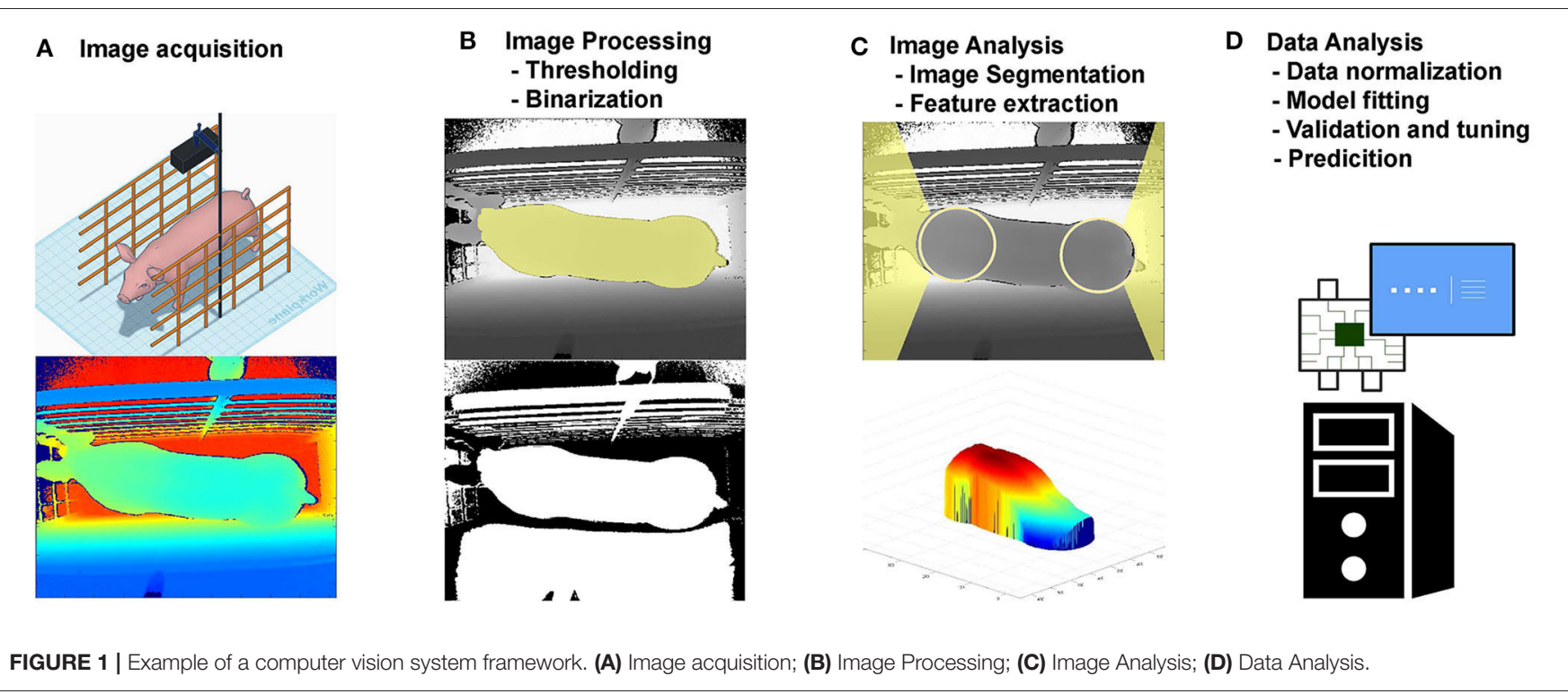

the development of mathematical methods for the identification of simple geometrical structures such as lines and circles (3, 10) or key-point features that can be jointly used to identify more complex objects or patterns $(11,12)$. Machine Learning is also a broader field that is concerned with the development and application of algorithms for extracting information from the most diverse data sets (13), and several machine learning algorithms have been developed or adapted specifically for solving computer vision problems.

An example of CVS is presented in Figure 1, where a $3 \mathrm{D}$ camera is used to capture images from pigs [adapted from (14)]. In a standard pipeline, after these images have been captured, they are processed (Figure 1B) using common imaging processing techniques such as image thresholding and binarization. Using the processed image, features of interest are identified, such as the pig head and tail (Figure 1C), and are removed together with the image background. From the resultant image of the pigs back, several measures were taken (e.g., volume, area, height, and length). These measures leverage important information from the images evaluated and can be used then for the development and evaluation of predictive models (Figure 1D), such as prediction of body weight.

\section{Image Formation}

An important aspect of digital imaging is how the image itself is acquired since there are sensors better suited for different applications. Before images could be processed and analyzed in computers, there was the need to develop sensors able to recognize, measure, and digitalize luminous signals. It was in the 1970s, with the advent of the charge-coupled devices (CCD) sensors (15), that digital imaging was developed, and the interest in CVS appeared. Basically, in digital image formation, luminous signals are captured by the sensor, coded, and stored in arrays of data that can be interpreted and manipulated in computational algorithms (9). Thus, for a computer, an image is nothing more than numerical values in a structured array of data that codifies light and colors for each point in the image (Figure 2). This array can be a single matrix, where the values inside the matrix correspond to black or white (binary image) or different shades of gray (grayscale image). Also, it can be an array of 3 matrices in the case of color images (i.e., intensities of red, green, and blue, on the RGB color space) or even multiple matrices for hyperspectral images. Therefore, mathematical manipulations and statistics of an image were among the first studies developed in digital image analysis and processing.

Another turning point in the history of computer vision was the advent of personal digital cameras in the 1990s, reducing the costs and popularizing the process of capturing and analyzing digital images $(16,17)$. Since then, several applications of digital photography have appeared. This popularization of digital cameras is directly connected to the increasing volume of data (photos and videos) generated over the last few years in many fields due to the increasing number of computer vision applications to solve the most diverse problems. This increased interest in computer vision and related areas can be illustrated by the increasing number of publications in the last decade (Figure 3).

Some areas of recent interest in computer vision are object sensing, mapping, recognition, motion tracking, navigation, image segmentation, and scene interpretation. However, while humans and animals do most of these actions intuitively, the majority of the vision tasks are considered as difficult problems in computer science, and the algorithms available are prone to errors (9). Thus, many successful CVSs are the result of multidisciplinary approaches tailored for specific cases, for example, interactive segmentation (8), face detection based on image features (18), and machine learning methods for object detection and recognition, such as optical recognition (19) and image classification, such as classification of regions and cells of histopathological images (20). 
A

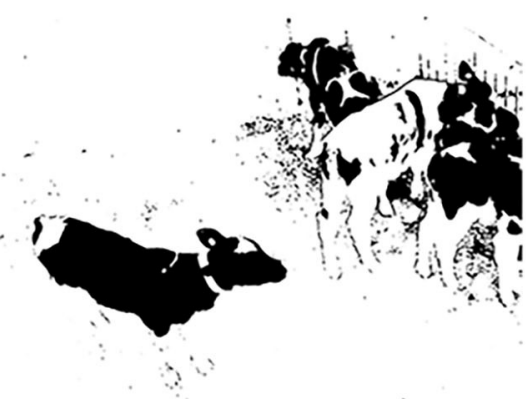

B

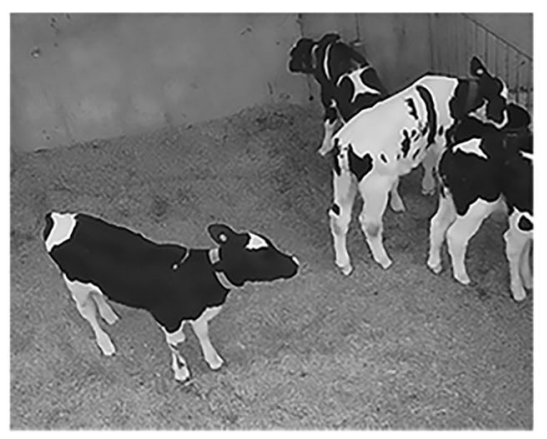

c

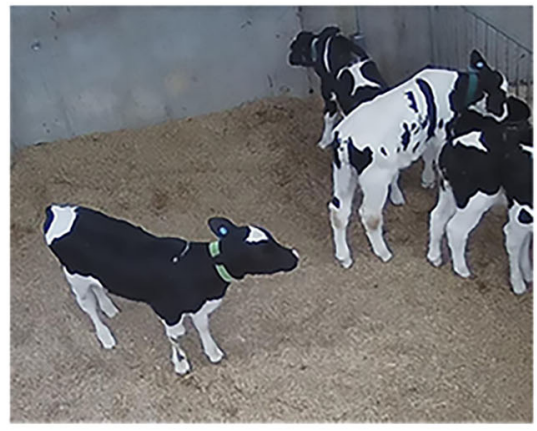

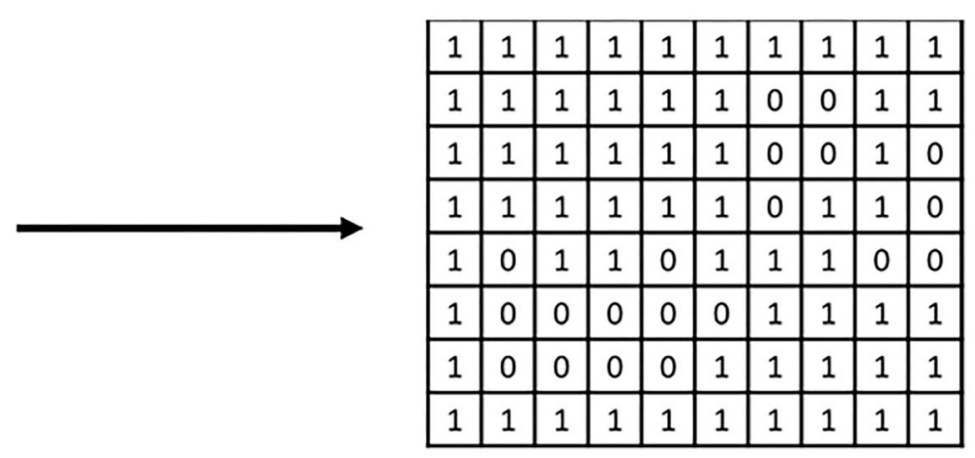

\begin{tabular}{|l|l|l|l|l|l|l|l|l|l|}
\hline 123 & 123 & 123 & 123 & 123 & 123 & 100 & 110 & 120 & 123 \\
\hline
\end{tabular}

\begin{tabular}{|l|l|l|l|l|l|l|l|l|l|}
123 & 123 & 123 & 123 & 130 & 120 & 35 & 30 & 100 & 110 \\
\hline
\end{tabular}
\begin{tabular}{|l|l|l|l|l|l|l|l|l|l|}
123 & 123 & 123 & 110 & 110 & 100 & 35 & 35 & 225 & 20 \\
\hline
\end{tabular}

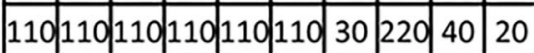
\begin{tabular}{|l|l|l|l|l|l|l|l|l|l|}
110 & 30 & 120 & 120 & 29 & 123 & 211 & 225 & 40 & 30 \\
\hline
\end{tabular}

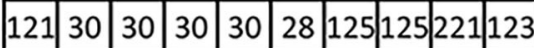
\begin{tabular}{|l|l|l|l|l|l|l|l|l|l|}
150 & 30 & 30 & 30 & 30 & 111 & 111 & 111 & 123 & 123 \\
\hline
\end{tabular}

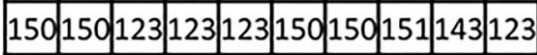

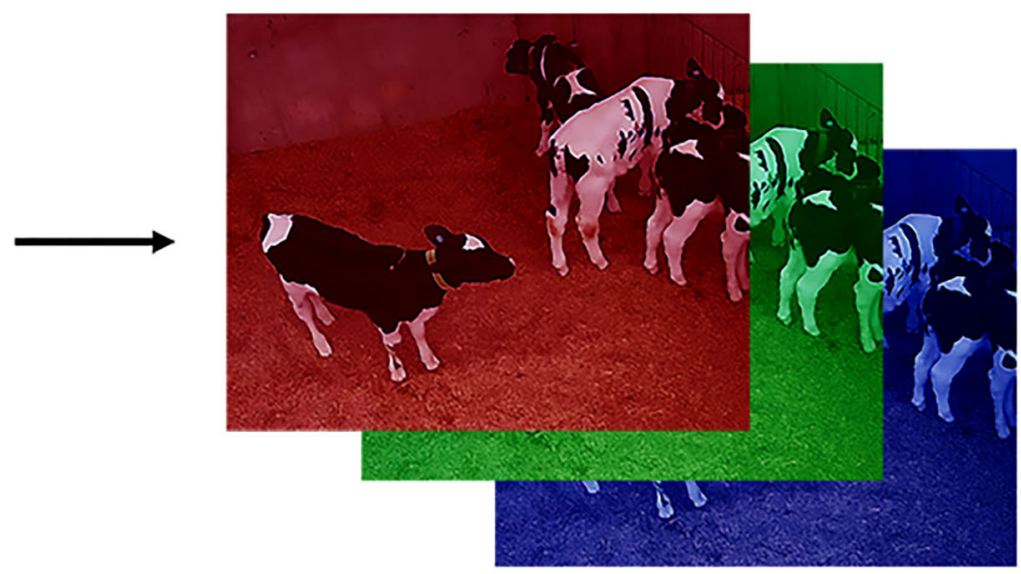

FIGURE 2 | Digital image representation. (A) Logical image with values 1 for white and 0 for black; (B) Grayscale image in the 24-bits depth format (values ranging from 0 to 255); (C) Color image on the RGB color space where each matrix is a 24-bits depth image, one for each color layer.

Among the machine learning techniques used in computer vision, it is worth mentioning deep learning algorithms, which have recently been successfully used in diverse computer vision applications. These algorithms are an extension of traditional artificial neural networks (ANN), and they achieve great power and flexibility by learning more abstract representations of the inputs as a nested hierarchy of concepts (21). These nested concepts, or hidden layers, generate very complex models with many parameters that were possible to be trained only with the advent of very large datasets, data augmentation techniques, and advancements in ANN, such as the development of learning optimization via stochastic gradient descent, new activation functions such as rectified linear unit (ReLU), regularization techniques, and efficient use of graphics processing unit (GPU) $(21,22)$.

\section{Metrics for Model Comparison and Assessing Predictive Ability}

As there are CVS developed for many different tasks and using a wide range of methods and models, there will be also many ways to compare competing approaches for different applications. In the following, we discuss some of these comparison methods by splitting them according to the class of the variable being predicted. For the scope of this study, we will split the predicted variable into two classes: (1) a variable that we deem associated/correlated to the image, and (2) the image per see (or even parts of the image). 


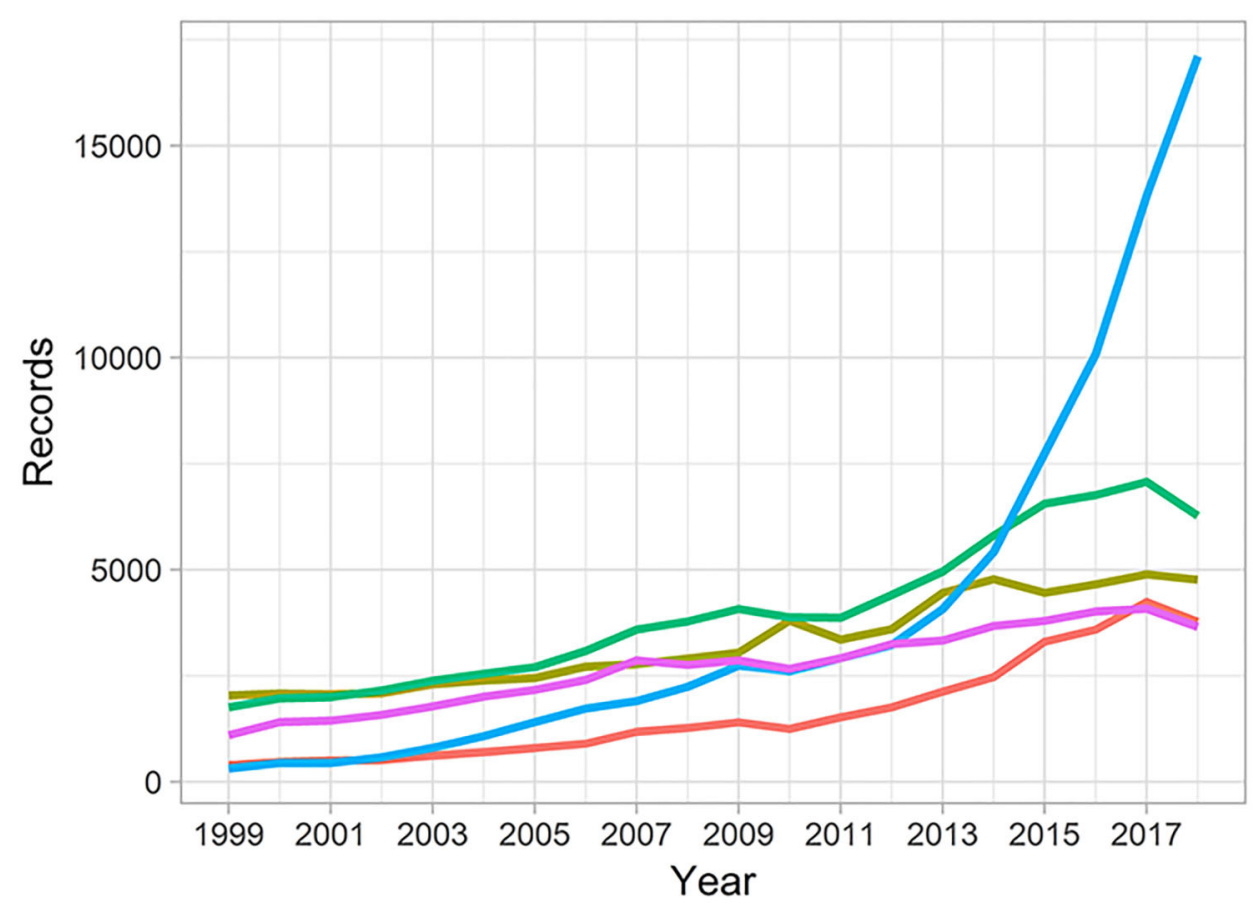

Field

- Computer Vision

- Image Analysis

Image Processing

Machine Learning

Pattern Recognition

FIGURE 3 | Count of publications hits in "Web of Science" for computer vision, image analysis, image processing, machine learning and pattern recognition.

In some applications, the interest will be to predict a variable by using information extracted from the images. These variables of interest can be a categorical variable such as animal species, behavior classes, and scores (e.g., leg score, body conditioning score), or a continuous variable such as body area, height, and weight.

For categorical variables, the simplest case assumes only two states (e.g., health/disease, moving/standing, among others), and the general scenario allows for multiple classes to be evaluated at the same time (e.g., behaviors such as laying, drinking, eating, walking, etc.). The metrics used to evaluate the predictive methods, in this case, are going to assess the frequency of two types of error: false positive (a.k.a. nuisance alarm) and false negative (a.k.a. missing alarm) errors and the most basic assessment tools are via tables of errors, or confusion matrix as below:

\begin{tabular}{lcc}
\hline & $\mathbf{y}=\mathbf{0}$ & $\mathbf{y}=\mathbf{1}$ \\
\hline$\hat{y}=0$ & TP & FP \\
$\hat{y}=1$ & FN & TN \\
\hline
\end{tabular}

Here, $y$ corresponds to the true category or ground truth (e.g., $y$ $=1$ for disease and $y=0$ for healthy), which can be a manual measurement or derived from a gold standard method, and $\hat{y}$ corresponds to the predicted class. The combination of each value of $y$ and $\hat{y}$ gives either a true positive (TP), true negative (TN), false negative (FN), or false positive (FP). From the confusion matrix and the TP, TN, FN, and FP counts for any given experiment, several metrics can be derived, such as: sensitivity (recall or true positive ratio $)=\frac{\mathrm{TP}}{\mathrm{TP}+\mathrm{FN}}$; false positive rate $(\mathrm{FPR})$, also known as, false discovery rate $(\mathrm{FDR})=\frac{\mathrm{FP}}{\mathrm{TP}+\mathrm{FP}}$; precision $=\frac{\mathrm{TP}}{\mathrm{TP}+\mathrm{FP}}$; specificity $=\frac{\mathrm{TN}}{\mathrm{TN}+\mathrm{FP}}$; and accuracy $=\frac{\mathrm{TP}+\mathrm{TN}}{\mathrm{TP}+\mathrm{FP}+\mathrm{TN}+\mathrm{FN}}$. However, when evaluating classification methods sometimes it is interesting to evaluate many threshold values used to classify $\hat{y}$ as one class or another. This evaluation is often done using receiver operating characteristic (ROC) curves, which measures the tradeoff between sensitivity and FDR, or 1- specificity (which yields the same value). Another metric is the precision-recall (PR) curve which is useful to evaluate the trade-off between precision and recall as the threshold value varies, i.e., the trade-off between the fraction of the detection that is actually true positives (i.e., precision) and the fraction of true positives that are detected (i.e., sensitivity) (13). Also, PR curves are especially interesting when we have situations with unbalanced data. In these situations, a ROC curve may present a misleading high area under the curve (AUC), for a model that is only predicting every data point as from the class that has more true values (Figure 4). For both curves, the quality of competing methods is often summarized by the AUC for which higher area means better fitting, with an area of one meaning a perfect fit.

For applications where the variable of interest is continuous, the predictive ability is typically evaluated using the Pearson product-moment correlation coefficient ( $r)$ between the input $(y)$ and the predicted output $(\hat{y})$ : 

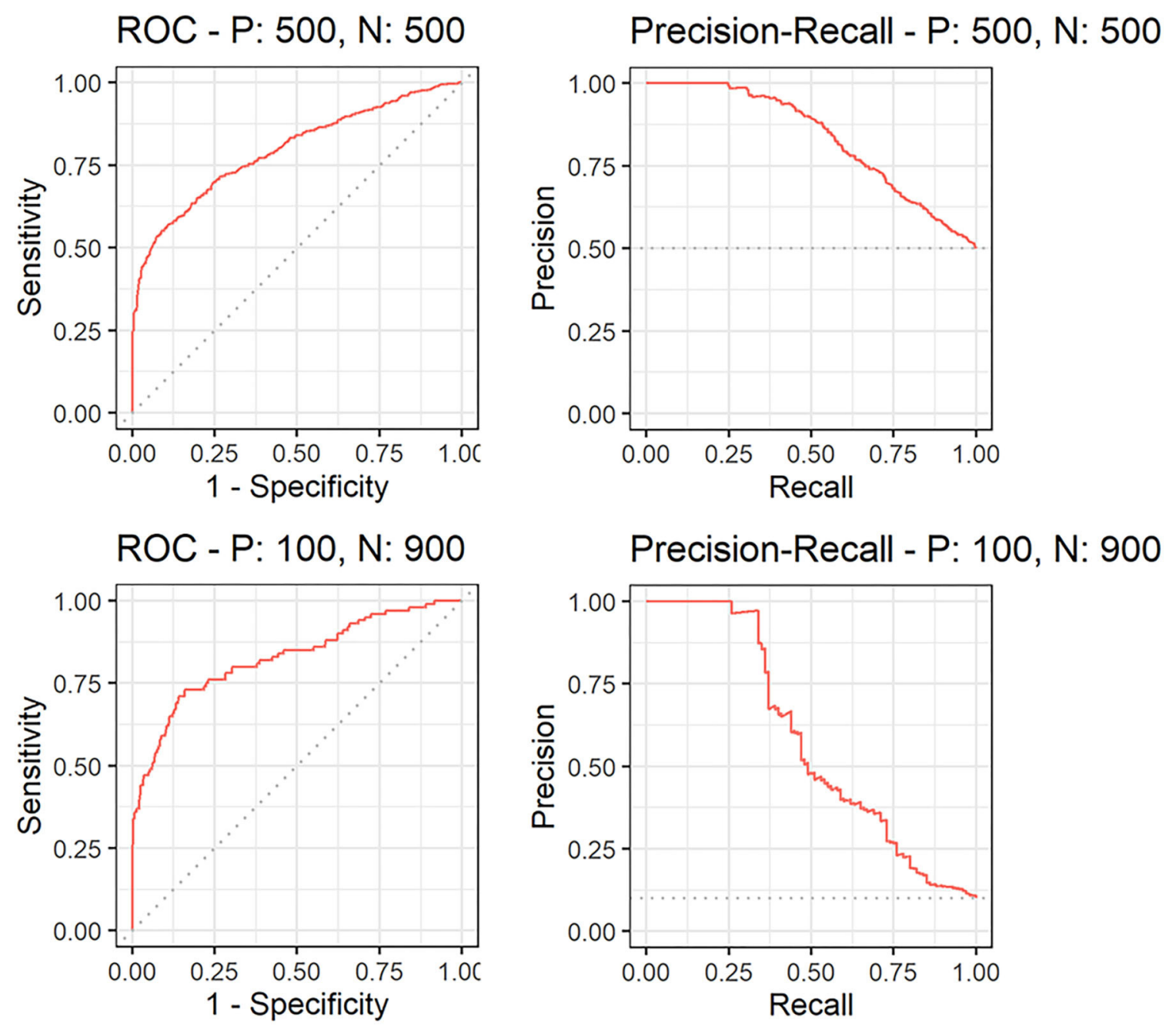

FIGURE 4 | Comparison of receiver operator curves (ROC) and precision-recall curves for a balanced [with 500 positive (P) and 500 negative (N) labels] and unbalanced (with $100 \mathrm{P}$ and $1102900 \mathrm{~N}$ ) datasets.

$$
\mathrm{r}==\frac{\operatorname{cov}(y, \hat{y})}{\sigma_{y} \sigma_{\hat{y}}}
$$

where $\operatorname{cov}(y, \hat{y})$ is the covariance, and $\sigma_{y}$ and $\sigma_{\hat{y}}$ are the square root of the input and output variances. Alternatively, instead of the predictive correlation $r$, its square is often reported. Both $r$ and $\mathrm{r}^{2}$ measure the linear relationship between $y$ and $\hat{y}$, and the closer to 1 the better. However, they are not measurements of prediction accuracy, as they do not take prediction bias into account. In this context, another measure often reported is the predictive error, or rather, the mean absolute predictive error (MAE or MAPE), which is a direct measure of how much the predictions deviate from the true values and can be defined as:

$$
M A E=\operatorname{mean}(|y-\hat{y}|)
$$

Because MAE can be influenced by the scale, when comparing between different studies it is often better to use a scale independent measure (23), such as the mean absolute scaled error (MASE), which can be defined as:

$$
\text { MASE }=\operatorname{mean}\left(\left|\frac{y-\hat{y}}{\operatorname{mean}(y)}\right|\right)
$$

In applications where the variable of interest can be the whole images or parts of the image, such as identification of objects within the image, the predictive ability can be evaluated via the pixel-wise accuracy, i.e., the ratio of pixels correctly predicted vs. the total number of pixels. However, this measure of prediction quality will tend to be high for most of the applications as the majority of the pixels within large objects will be correctly predicted. Another interesting measure in this scenario is the Jaccard index, a.k.a. Intersection over Union (IoU), which is the ratio of the intersection between the ground truth $(A)$ and the predicted area $(\hat{A})$ by the union of these areas: 


$$
\operatorname{IoU}_{(A, \hat{A})}=\frac{|A \cap \hat{A}|}{|A \cup \hat{A}|}
$$

Thus, IoU is a measure of similarity between the two areas, and values closer to 1 indicate more similarity, meaning a better fit of the predictive method (24).

As a final note on this topic, it is important to evaluate the generalization performance of the candidate methods, in other words, their predictive capability on independent data set. This evaluation will provide insight into the variability of the predictive error as well as potential overfitting (a situation when a method performs very well on the training data but not so on the independent dataset). This independent dataset, or validation set, is ideally a dataset collected in another moment from different animals. But most of the time if the data is large enough it can be a reserved portion of the original data. Another interesting technique is cross-validation, where the data is divided into multiple subsets, and each time one of the subsets is reserved for validation while the others are used for training. Thus, in a $\mathrm{k}$-fold cross-validation, the dataset is divided into $\mathrm{k}$ subsets, and if $k=n$ (number of data points) the approach is called a leaveone-out cross-validation. For a more in-depth reading on model assessment and selection, the reader can refer to Chapter 7 of Hastie et al. (25).

\section{SENSORS USED FOR IMAGING IN ANIMAL AND VETERINARY SCIENCES}

Currently, the most used image sensor devices are standard digital cameras and/or surveillance cameras that capture electromagnetic waves within the visible light spectra to generate digital images (color or grayscale). However, there are also other technologies that have been used for more specific applications, such as devices that are based on infra-red, ultrasound, and ionizing radiation. Moreover, some technologies can generate more complex arrays of images such as threedimensional (3D) and hyperspectral images. They are, however, in general, more expensive than standard digital cameras. Nonetheless, each different imaging technology can be used for specific applications.

\section{Images on the Visible Light Spectrum}

Cameras for standard digital imaging work with signals within the visible light range, and they generally have a CCD or a complementary metal-oxide-semiconductor (CMOS) sensor. Both sensors have a similar function, which is to capture light and convert it into a digital image, however, they have some important differences. On one hand, CCD sensors are charged passively by the light source, and the information captured in each pixel is processed sequentially. CMOS sensors, on the other hand, have active pixels with a transistor for each pixel so that the information from each pixel is translated to the image independently and generally asynchronously to the digital image (26). These differences in sensor architecture lead to differences in sensor prices and capabilities. Even though the industry is in constant development, CCD sensors, in general, have a higher dynamic range and produce more uniform images, while CMOS sensors are cheaper, energy efficient, and more responsive.

\section{Infrared Radiation}

Infrared radiation (IR) has a wavelength longer than the visible light, and according to the International Organization for Standardization (ISO) it can be divided into near-infrared (NIR), mid-infrared (MIR), and far-infrared (FIR). This division has been based on the specific wavelength thresholds of $0.78-3,3-50$, and $50-1,000 \mu \mathrm{m}$ for NIR, MIR, and FIR, respectively. There are many different applications of IR in imaging, and for the purpose of this review, the most significant ones are in 3D imaging, spectroscopy, night vision, and thermal imaging (also known as Thermography). For all these applications, there are different IR sensors specific to capture radiation within NIR, MIR, or FIR ranges. In most of the night vision cameras, the sensors rely on an emitter, which emits IR on the NIR wavelength to actively illuminate the scene. On the other hand, thermal imaging uses the principle that all objects produce radiant heat (emitted, transmitted, and/or reflected), thus there is no need for an emitter since the sensors are capable of capturing the heat signal in the MIR or FIR range (27). The sensors for thermal imaging can be divided into two groups, cooled or uncooled focal plane array. The main difference is that the cooled sensors generally produce better images and less variable measurements, at the cost of being heavier, less portable, and expensive.

In animal, veterinary, and wildlife applications, both night vision and thermal cameras have been used mostly for monitoring animals (livestock or wildlife) at night or dim light situations, either alone or in association with standard digital image sensors (28). Such applications can be dated to the use of military night vision scopes for observation of nighttime animal behavior in the 70s (29). However, thermal cameras also have applications in diagnostic imaging to detect small changes in the body's surface temperature (30), which can be due to stress, fever, inflammation, and ischemia. Nevertheless, proper use of thermal imaging for diagnostic purposes still requires correct calibration of the device, adequate location, and correct positioning of the animal and camera $(27,30)$.

\section{D Imaging}

Many different sensors and techniques are used for measurement of the distance of objects to the camera, acquisition, and formation of $3 \mathrm{D}$ images. In livestock, these sensors can be used, for example, for measurement of animal volume, surface, and gait, among other traits. From the several technologies developed for 3D imaging, we will focus on optical applications (i.e., applications that use radiation on visible and near-visible light) used in 3D cameras, also known as depth sensors. These techniques can be further divided into passive, such as stereo imaging and structure from motion, or active, such as structured light and time of flight (31).

In stereo imaging, two or more cameras are used, and principles of epipolar geometry are applied in order to calculate the distance $(z)$ of a point P to the cameras (32). In a simplified stereoscopic triangulation (Figure 5) using two similar cameras with the same focal length $f$, the differences of the projections 


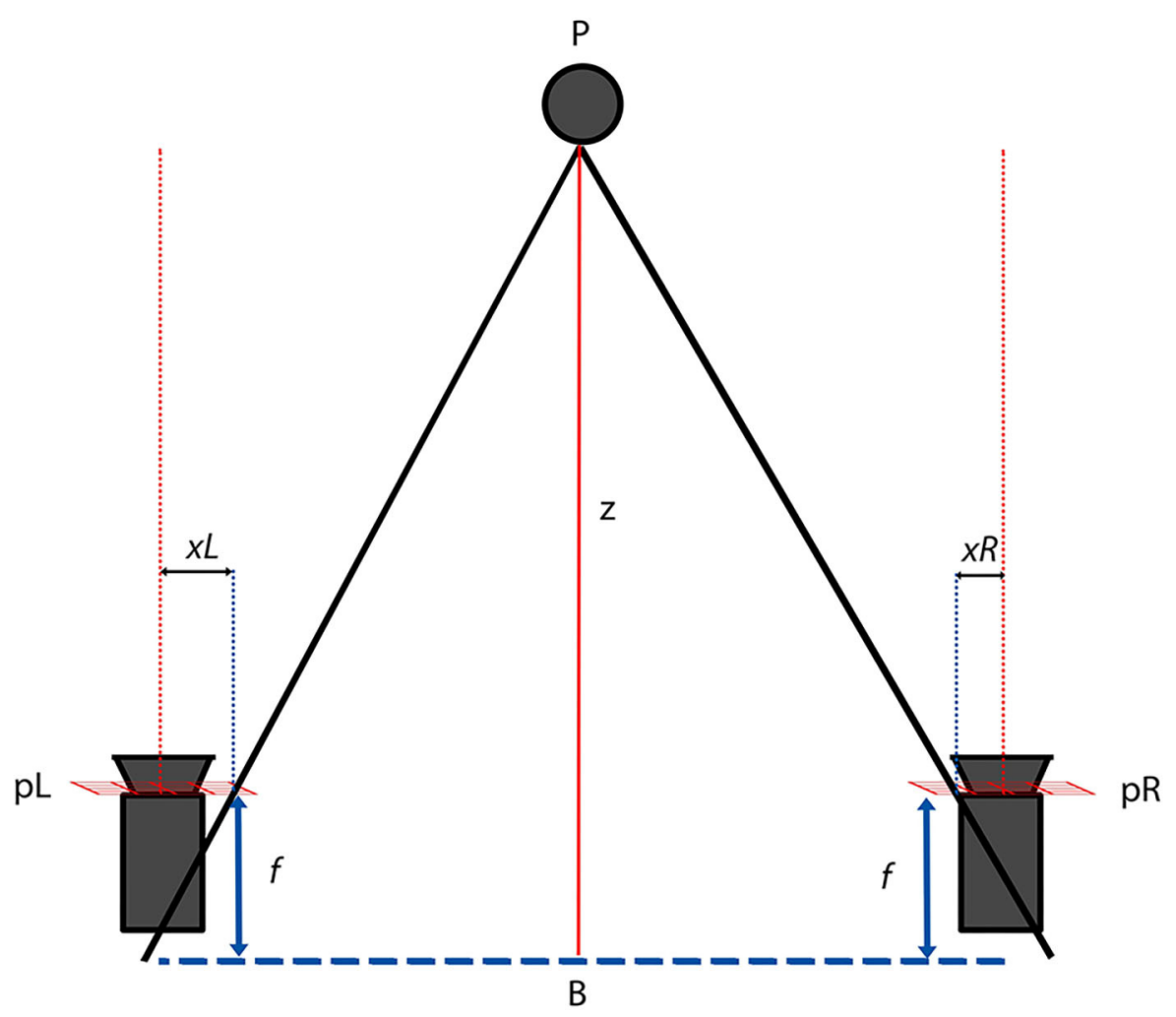

FIGURE 5 | Epipolar triangulation used on a rectified stereo imaging system with two similar cameras. $\mathrm{P}$ is the point of interest; $\mathrm{xL}$ and $\mathrm{xR}$ its projection on the camera planes $\mathrm{pL}$ and $\mathrm{pR}$; $\mathrm{f}$ is the camera focal length and $\mathrm{B}$ the baseline plane.

$\left(x_{L}\right.$ and $\left.x_{R}\right)$ of point $\mathrm{P}$ on the planes of the cameras is the disparity between the images. That disparity can be used then to calculate the distance of point $\mathrm{P}$ to the baseline plane, where $\mathrm{B}$ is the distance between cameras.

Similarly, in structure from motion, a disparity map can be created between the images from a single moving camera, in which case the distance between the points where each image was captured by the camera can be used as the distance between "cameras." The main difficulty of such a strategy is that it needs the object of interest to be practically motionless.

Structured light, also known as coded light refers to the use of active emission of known light patterns for which the illuminated surface will present structural distortions in the shade/light patterns according to irregularities in the surface, angle, and distance to the emitter (Figure 6). Therefore, similarly to stereo imaging, the distortions in the emitted patterns provide unique correspondence for triangulation with the camera. The emitter can vary from a punctual laser, a blade scanner, multiple shadow patterns that split the scene into areas of interest, or even the use of complex multi-laser patterns that create a spatial neighborhood $(31,32)$.

Time of flight (ToF) and Light Detection and Ranging (LiDAR) cameras are based on signal modulation and ranging, similar to other technologies such as Sound Navigation Ranging (SONAR) and Radio Detection and Ranging (RADAR) (32). These techniques measure the distance between the sensor and a target object by detecting the time difference from the signal emitted by a transmitter, reflected on a target object, and captured back by a receiver (Figure 7). Even though the principle is simple, there is a great implementation challenge due to the speed of light, interference with natural light, dispersion, and absorption of the light. Modern ToF cameras generally consist of a transmitter array that emits a modulated IR or NIR light (to reduce environmental interference) and a receiver array that captures the reflected signal and calculates the signal phase/time lag for each pixel (31). Recently, in order to improve the transmitter performance, devices equipped with micro-electromechanical system (MEMS) mirrors have received great interest from the scientific and industry community (33).

Lastly, there are also hybrid 3D cameras that combine RGB sensors with depth sensors based on one or more of the technologies described above. Examples include cameras based on active stereoscopy, which combine stereo imaging from multiple cameras with structured light to improve the depth estimation. Table 1 shows some of the current 3D cameras available and their technical specifications.

\section{Other Imaging Technologies of Importance}

There are many other imaging related technologies of importance in animal and veterinary sciences, such as spectral and hyperspectral imaging, ultrasound, $\mathrm{x}$-ray, computed 


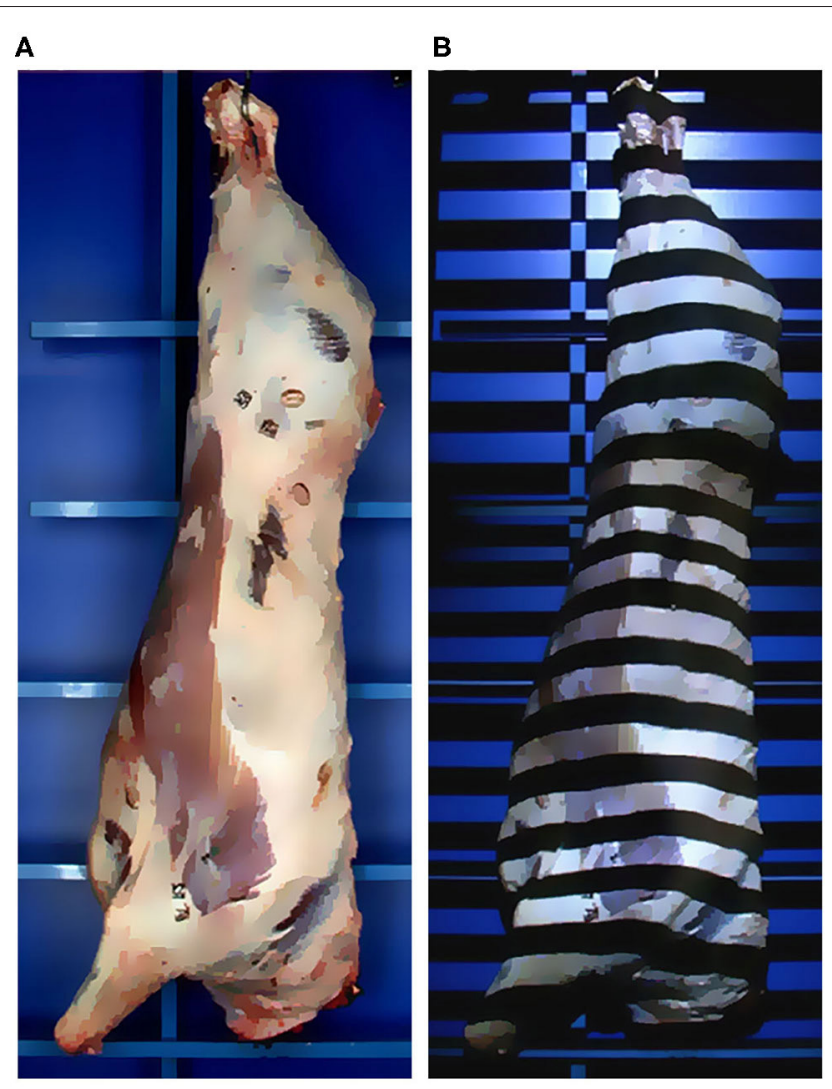

FIGURE 6 | Example of a structured light system based on linear shadow pattern. (A) The scene with natural illumination. (B) The same scene now under the structured light projected by the emitter.

tomography, and satellite images. In the following, we briefly describe some of these technologies.

Spectroscopy, spectral imaging, and more recently hyperspectral imaging have been adopted extensively for evaluation of meat attributes and chemical characteristics as well for quantification of milk protein and fat content $(34,35)$. These technologies are mostly composed of sensors equipped with a NIR emitter, and are based on the principle that different compounds will absorb the radiation differently in each wavelength, thus generating a "signature" (36). In Spectroscopy, for each wavelength measured inside the range, a punctual value of absorbance is generated. Meanwhile, a spectral image is a matrix with a value of absorbance for each pixel, and a hyperspectral image corresponds to a cube of several matrices (one for each wavelength) providing both spectral and spatial information.

Several medical diagnostic imaging technologies have also been employed for many applications with animals such as evaluation of muscle and fat composition, and bone mineralization in live animals and carcasses (37-39). Some worth noting technologies include ultrasound (US), dual-energy $\mathrm{x}$ ray absorptiometry (DXA), computed tomography (CT), and magnetic resonance (MR). All these technologies are appealing since they make possible the generation of images for the evaluation of the body composition. From these technologies, only US is currently used in farm conditions due to many factors such as price, portability, and no anesthesia required. Nonetheless, it still requires a trained operator.

\section{APPLICATIONS OF COMPUTER VISION SYSTEMS IN ANIMAL AND VETERINARY SCIENCES}

Before the advent of CVS, many applications required the use of the trained eye for visual classification of traits in live animals, such as animal behavior, body condition score, carcass fat deposition, meat marbling, or classification of eggshell quality. There are also methods that use the aid of lenses, such as microscopes, for evaluation of cell morphology in a blood smear or spermatozoid motility and defects. Moreover, other signals such as ultrasound, infrared, and x-rays are widely used to produce images for diagnostic purposes. However, most of the methods currently used for the measurement of traits of interest need expert personnel requiring the training of evaluators from time to time to maintain good measurement quality. Also, most of such measuring processes are time demanding, stressful to the animals, and costly for the farmer, making it prohibitive due to animal welfare and economic reasons. Therefore, there is an interest in developing automatic, indirect methods for monitoring livestock and measuring traits of interest. For such tasks, CVS generally uses algorithms and principles of pattern recognition, image analysis, and processing in order to tackle the most diverse problems. The framework presented in Figure 1 can be seen as a CVS pipeline, with a fixed sensor capturing the information that is presented by the world or actively exploring the world and adjusting its perception (field of view, exposure, among others). The development of automated CVS can enable high-throughput phenotyping in livestock, and the data generated by such systems can be then used for many different applications, from the development of smart farm management tools to advancing breeding programs.

In the following, we present selected applications of CVS as an answer to the need for such automated, non-invasive methods for the measurement of carcass and meat traits, live animals' identification, tracking, monitoring, and phenotyping using different sensors.

\section{Carcass and Meat Traits}

Probably one of the first applications of a CVS was in meat sciences, with the earliest reported studies found in the 1980s (40-42). In these studies, the system was composed of a camera, light source, digitizer, and computer unit. The CVS needed an operator to position beef meat cuts on a surface at a known distance and angle from the camera, and to trigger the image acquisition. Thus, the meat cuts were all positioned in the same manner with constant background and illumination. At that time, the interest was to predict the cut content of lean meat and fat, and to compare the results from the CVS to trained United States Department of Agriculture (USDA) meat graders. 


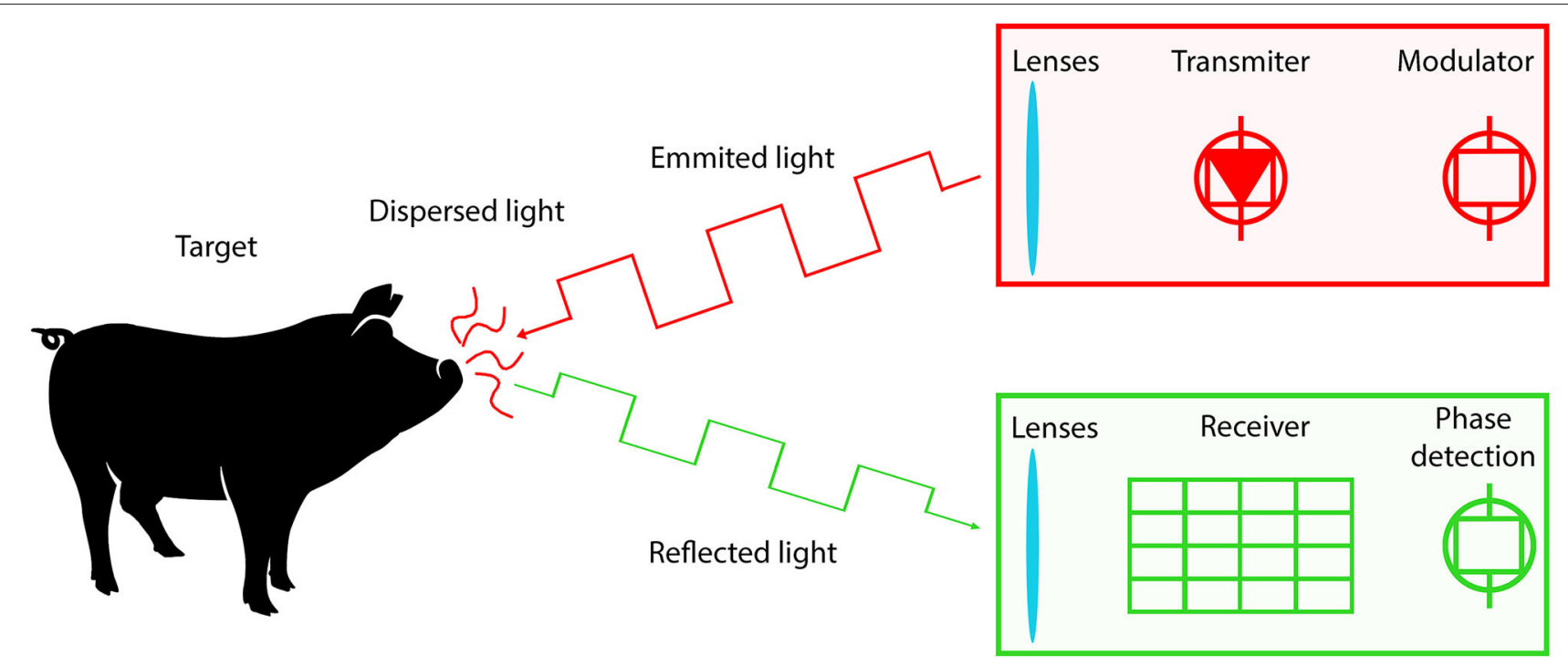

FIGURE 7 | Principle of time of flight (ToF) 3D cameras (depth sensors).

TABLE 1 | Comparison of 3D cameras and their technical specifications.

\begin{tabular}{|c|c|c|c|c|c|c|c|c|}
\hline Device & Manufacturer & Sensors & Technology & Range (m) & Environment & FPS & FOV $(\mathrm{V} \times \mathrm{H})$ & $\begin{array}{l}\text { Resolution } \\
\text { (pixels) }\end{array}$ \\
\hline Kinect V1 ${ }^{\mathrm{a}}$ & Microsoft & $\begin{array}{l}\text { 3D (IR emitter + IR camera) } \\
\text { Color }\end{array}$ & Structured Light & $0.8-4$ & Indoor & $\begin{array}{l}30 \\
30\end{array}$ & $45^{\circ} \times 58^{\circ}$ & $480 \times 640$ \\
\hline Kinect V2 ${ }^{\mathrm{a}}$ & Microsoft & $\begin{array}{l}\text { 3D (IR emitter + IR camera) } \\
\text { Color }\end{array}$ & Time of flight & $0.5-4.5$ & Indoor & $\begin{array}{l}30 \\
30\end{array}$ & $\begin{array}{l}60^{\circ} \times 70^{\circ} \\
54^{\circ} \times 84^{\circ}\end{array}$ & $\begin{array}{c}424 \times 515 \\
1080 \times 1920\end{array}$ \\
\hline Kinect Azure & Microsoft & $\begin{array}{l}\text { 3D-N (IR emitter + IR camera) } \\
\text { 3D-W (IR emitter + IR camera) } \\
\text { Color }\end{array}$ & $\begin{array}{l}\text { Time of flight } \\
\text { Time of flight }\end{array}$ & $\begin{array}{l}0.5-5.5 \\
0.3-2.8\end{array}$ & Indoor & $\begin{array}{l}30 \\
30\end{array}$ & $\begin{aligned} 65^{\circ} & \times 75^{\circ} \\
120^{\circ} & \times 120^{\circ} \\
59^{\circ} & \times 90^{\circ}\end{aligned}$ & $\begin{array}{c}576 \times 640 \\
1024 \times 1024 \\
2160 \times 3840\end{array}$ \\
\hline Xtion ${ }^{a}$ & Asus & $3 D$ & Time of flight & $0.8-3.5$ & Indoor & 30 & $45^{\circ} \times 58^{\circ}$ & $480 \times 640$ \\
\hline Xtion Pro Live & Asus & $\begin{array}{l}3 \mathrm{D} \\
\text { Color }\end{array}$ & Time of flight & $0.8-3.5$ & Indoor & $\begin{array}{l}30 \\
30\end{array}$ & $45^{\circ} \times 58^{\circ}$ & $\begin{array}{c}480 \times 640 \\
1024 \times 1280\end{array}$ \\
\hline Xtion 2 & Asus & $\begin{array}{l}3 \mathrm{D} \\
\text { Color }\end{array}$ & Time of flight & $0.8-3.5$ & Indoor & $\begin{array}{l}30 \\
15\end{array}$ & $\begin{array}{l}52^{\circ} \times 74^{\circ} \\
60^{\circ} \times 75^{\circ}\end{array}$ & $\begin{array}{c}480 \times 640 \\
1944 \times 2592\end{array}$ \\
\hline Intel D435 & Intel & $\begin{array}{l}\text { 3D (IR emitter + IR camera) } \\
\text { Color }\end{array}$ & Active Stereo & $0.1-10$ & indoor/outdoor & $\begin{array}{l}90 \\
30\end{array}$ & $\begin{array}{l}58^{\circ} \times 87^{\circ} \\
43^{\circ} \times 70^{\circ}\end{array}$ & $\begin{array}{r}720 \times 1280 \\
1080 \times 1920\end{array}$ \\
\hline Intel L515 & Intel & $\begin{array}{l}\left.\text { 3D (IR emitter + MEMS }{ }^{\mathrm{b}}\right) \\
\text { Color }\end{array}$ & LIDAR & $0.2-9.0$ & Indoor & $\begin{array}{l}30 \\
30\end{array}$ & $\begin{array}{l}55^{\circ} \times 70^{\circ} \\
43^{\circ} \times 70^{\circ}\end{array}$ & $\begin{array}{c}768 \times 1024 \\
1080 \times 1920\end{array}$ \\
\hline Structure ${ }^{a}$ & Occipital & 3D (IR emitter + IR camera) & Structured Light & $0.8-4.0$ & Indoor & 30 & $43^{\circ} \times 57^{\circ}$ & $480 \times 640$ \\
\hline Structure II & Occipital & $\begin{array}{l}\text { 3D (IR emitter + IR camera) } \\
\text { Ultra-wide monochrome }\end{array}$ & Active Stereo & $0.3-5.0$ & indoor/outdoor & $\begin{array}{c}54 \\
100\end{array}$ & $\begin{array}{l}46^{\circ} \times 59^{\circ} \\
160^{\circ} \text { Diag }\end{array}$ & $\begin{array}{l}960 \times 1280 \\
480 \times 640\end{array}$ \\
\hline Structure Core & Occipital & $\begin{array}{l}\text { 3D (IR emitter + IR camera) } \\
\text { Color }\end{array}$ & Active Stereo & $0.3-10$ & indoor/outdoor & $\begin{array}{c}54 \\
100\end{array}$ & $\begin{array}{l}46^{\circ} \times 59^{\circ} \\
85^{\circ} \text { Diag }\end{array}$ & $\begin{array}{c}960 \times 1280 \\
480 \times 640\end{array}$ \\
\hline
\end{tabular}

${ }^{a}$ Out of production/Discontinued; b Micro-electro-mechanical system mirror.

In these studies, multiple linear regressions fitting variables extracted from the CVS were compared to models that included manual measurements and USDA graded variables. Even though the system was not fully automated, the prediction equations developed with the variables measured by the system presented slightly better results than the prediction equations developed with variables measured by trained graders. The best linear model for prediction of lean meat weight developed with the variables 
TABLE 2 | Examples of computer vision applications in meat sciences (studies highlighted in bold were with live animals).

\begin{tabular}{|c|c|c|c|}
\hline & Applications & Image signal & References \\
\hline \multirow{4}{*}{$\begin{array}{l}\text { Cattle and Small } \\
\text { Ruminants }\end{array}$} & Carcass & 3D; US; VL & $(41,43,44)$ \\
\hline & Fat (kg and\%) & US, VL & $(41,43,45)$ \\
\hline & Lean meat (kg and \%) & VL & $(41,42,45)$ \\
\hline & Tenderness & VL & $(45,46)$ \\
\hline \multirow[t]{3}{*}{ Fishery } & $\begin{array}{l}\text { Fat } \\
\text { Pigmentation }\end{array}$ & $\mathrm{IR} ; \mathrm{VL}$ & $(47)$ \\
\hline & Sorting & HS & $(48)$ \\
\hline & Freshness & VL; HS; 3D & $(49,50)$ \\
\hline \multirow[t]{3}{*}{ Poultry } & Classification & HS; VL & (51) \\
\hline & Brest weight & $3 D$ & (52) \\
\hline & Egg shell classification & VL & (53) \\
\hline \multirow[t]{3}{*}{ Pork } & Carcass & US; VL; CT; 3D & $(37,39,54-56)$ \\
\hline & Classification & HS; VL & (51) \\
\hline & Quality & HS; IR; VL & $(57)$ \\
\hline
\end{tabular}

CT, Computed Tomography; 3D, 3-dimensional; HS, Hyperspectral; IR, Infrared; US, Ultrasound; VL, Visible Light.

extracted from the CVS achieved $R^{2}$ from 0.93 to 0.95 against $0.84-0.94$ from the model that included USDA graded covariates $(41,42)$.

Since these earlier studies, there has been an increasing interest in the use of computer vision for prediction of the most diverse meat quality traits, not only for beef but also for fish, poultry, and pork (Table 2). There are applications focused on imaging technologies for determination of not only meat crude protein and fat content but also more refined chemical characteristics like fatty acids profile, freshness $(50,57)$, as well as prediction of meat quality, palatability, tenderness, and other traits normally evaluated by a panel of trained experts $(45,46,51)$ or even automatic sorting and weighing cuts and viscera which is normally performed manually $(48,52)$. Again, different devices and imaging technologies have been used, with several predictive approaches evaluated. However, independently of the imaging device used, such applications pipelines generally involve several steps such as: (1) Sample preparation with standardization of meat cut used, presentation, background and light conditions; (2) Device calibration (when needed), collection and processing of the images; (3) Direct measurement of attributes of interest using a gold standard methodology (i.e., chemical analysis); and (4) Model fitting, which corresponds to the prediction of the gold standard using the image features as predictors. It is interesting to note that the image processing in step 2 can involve several sub-steps such as histogram equalization, background removal, and image smoothing. Also, in the case of hyperspectral images the processing involves selection of wavelengths and/or reduction of dimensionality using techniques such as Fourier transformation (58) and principal components, for an in-depth review of applications of hyperspectral imaging see Xiong et al. (34). It is also worth noting, that there is no standard model of choice for step 4 since in the literature several predictive approaches have been evaluated. These predictive approaches could be statistical models such as linear and partial least square regression to machine learning methods, such as support vector machines, random forests, and artificial neural networks.

\section{Monitoring and Phenotyping of Live Animals}

Differently from carcass and meat cuts that can be easily positioned for image acquisition under a well-controlled light source and even background, several obstacles arise when working with live animals. As an example, in farm conditions, the illumination can change throughout the day even inside a barn due to sun position, clouds, and seasons. Moreover, there will be differences between artificial light sources from one farm/barn to another, as they may use different types of lamps with different voltage and positioning. The background is also going to be different in each location, and it is prone to changes over time for a given location. Examples of differences in the background are floor surface material for animals in a barn and vegetation for free-range animals. Therefore, the diversity of situations is probably one of the biggest challenges in implementing a CVS that are robust enough to perform satisfactorily across different farm conditions.

Nevertheless, many efforts have been made over the years to develop CVS for monitoring and phenotyping livestock, poultry, and fish as well. In the current study, we do not intend to deliver an extensive review on the matter as there are already reviews on technology applications for poultry $(59,60)$, machine vision for detection of cattle and pig behavior (61), and computer vision applications for fisheries (62). Nonetheless, in the following, a broader view is presented regarding applications developed for traits of interest in animal and veterinary sciences, providing examples from earlier works to the current trends while highlighting challenges, advances that have been made, and areas of current interest. Table 3 shows a summary of selected applications, presenting the traits of interest and the kind of imaging sensor used.

\section{Evaluation of Body Composition, Meat, and Carcass Traits in Live Animals}

In section Carcass and Meat Traits we saw that CVS had been successfully used for prediction of traits such as lean meat and fat content from carcass and meat cuts. Nevertheless, the same predictive performance has not been observed initially for the evaluation of meat and carcass traits in live animals.

Initial attempts for the prediction of carcass composition on swine have been performed by Doeschl-Wilson et al. (54) where a CVS achieved a predictive $R^{2}$ of 0.31 and 0.19 for fat and of 0.04 and 0.18 for lean meat on the foreloin and hindloin regions, respectively. In the search for improvement of performance on the prediction of carcass and meat traits on live animals, researchers have focused on the use of medical imaging devices for such tasks. In recent studies using medical imaging devices, ultrasound measurements presented positive and moderate correlations of 0.6 and 0.56 with the carcass measurements of lean meat and fat depths, while CT presented low to moderate correlations of $0.48-0.67$ for fat and high correlations of $0.91-0.94$ for lean meat $(37,39)$. Moreover, these 
TABLE 3 | Examples of computer vision applications in live animals.

\begin{tabular}{|c|c|c|c|}
\hline & Applications & Image signal & References \\
\hline \multirow{7}{*}{$\begin{array}{l}\text { Cattle and small } \\
\text { ruminants }\end{array}$} & Mastitis & $\mathbb{R}$ & (63-66) \\
\hline & Digital dermatitis & $\mathbb{R}$ & $(67,68)$ \\
\hline & Body temperature & TR & $(69-71)$ \\
\hline & $\begin{array}{l}\text { Gait and body } \\
\text { measurements }\end{array}$ & $3 D$ & $(44,72)$ \\
\hline & Weight & $3 D$ & $(44,73)$ \\
\hline & $\begin{array}{l}\text { Coat and } \\
\text { conformation }\end{array}$ & VL & (74) \\
\hline & Body condition & VL; TR; 3D & (75-78) \\
\hline \multirow[t]{3}{*}{ Fishery } & Tracking & $3 D$ & (79) \\
\hline & Shape & $\mathrm{VL}$ & (80) \\
\hline & Weight & VL & (81) \\
\hline \multirow[t]{2}{*}{ Poultry } & Behavior & VL; 3D & (82-84) \\
\hline & Shape & $3 D$ & (84) \\
\hline Dog & Behavior & $3 D$ & (85) \\
\hline \multirow[t]{4}{*}{ Pork } & Tracking & $\mathrm{VL} ; 3 \mathrm{D}$ & (86-90) \\
\hline & Behavior & $\mathrm{VL} ; 3 \mathrm{D}$ & $(91-94)$ \\
\hline & Weight & $\mathrm{VL} ; 3 \mathrm{D}$ & $(14,95-97)$ \\
\hline & $\begin{array}{l}\text { Gait and body } \\
\text { measurements }\end{array}$ & $3 D$ & $(14,97-99)$ \\
\hline
\end{tabular}

3D, 3-dimensional; TR, Thermography; VL, Visible Light.

technologies have several drawbacks regarding animal handling and cost, as explained previously. In order to tackle these limitations, some recent works developed CVS based on 3D cameras for non-contact automated estimation of muscle score (55) and of fat and lean muscle content (56) on live pigs. Alsahaf et al. (55) developed a system that extracted morphometric features from the images of moving pigs for prediction of muscle scores between 1 and 5 . With a gradient boosted classifier, they achieved classification accuracy between 0.3 and 0.58 , and MAE of 0.65 , showing that most of the errors where between neighboring classes. Meanwhile, Fernandes et al. (56) evaluated not only features extracted from the images, but also deep learning methods that do not require image processing, with the deep learning approaches achieving better results. Their results present an improvement over previous studies with crossvalidation predictive $\mathrm{MAE}$, and $R^{2}$ for lean muscle depth of $3.33 \mathrm{~mm}$ and 0.50 , respectively, and of $0.84 \mathrm{~mm}$ and 0.45 for fat depth. Nevertheless, these $R^{2}$ presented are still low showing and there is room for improvement on the predictions of lean muscle and fat.

\section{Animal Tracking and Behavior Analysis Using CCD or CMOS Cameras}

Some of the most desired applications of CVS for live animals correspond to animal identification, tracking, and monitoring, ultimately identifying changes in their daily behavior. Animal identification can refer to the identification of an animal when there is only one animal in the image to more complex scenarios where there are multiple animals in the image or the identification of different individual animals in a single or multiple images matching their identification. Meanwhile, tracking involves the continuous identification of the animal across frames in a video feed or across images from different locations as the animals are moved from one location to the other. Regarding behavior, animals tend to synchronize their behavior within a group, and conspicuous deviations may be caused by environmental stress, management problems, or disease, although individual behavioral differences need to be taken into account. Therefore, there is a constant effort to understand behavioral changes and their relationship with other traits of interests, such as animal health status and growth. Closer evaluations of animal behavior and health are normally conducted by trained evaluators at specific time points, such as the time of transferring animals from one location to another (e.g., from nursery to grow-out farms) or around vaccinations. This is because managers and workers have limited time to spend in observing a group of animals. Also, with the current trend of an increasing size of livestock operations, there is also an increase in the animal/manager ratio. Thus, a basic use of CVS for evaluation of animal behavior can be the acquisition and storage of images and videos that can be assessed later or remotely by the farmers. This improves animal management since there is no need for the evaluator to be physically present, which otherwise can cause behavioral changes on the animals. Also, the evaluator can loop across images, and replay them, improving the quality of the evaluation. Nonetheless, this kind of system is not optimum since the evaluator would still need to check all the images. Therefore, there are efforts in the literature that attempt to develop CVS that can automatically classify animal behavior and alert the manager in real time regarding important changes.

Initial works with pigs demonstrated the applicability of a CVS to identify the animal position and to track its movement (86, 87). These works showed that the image processing algorithms available at the time could segment a single pig from the background under specific conditions. The conditions were: (1) camera positioned to get the top view of the animal, and (2) dark background for a white pig. The method developed by Tillett et al. (86) estimated a point distribution of landmarks on the pig contour for a sequence of frames (13-30 frames) and was able to model small changes in the animal's posture. However, only seven sequences where evaluated and it was prone to miss the animal if the changes in position were abrupt from one frame to another. On the other hand, Lind et al. (87) used a more robust segmentation approach based on the generation of a background matrix for image subtraction and consequently animal segmentation. Even though this method cannot identify animal posture, it was efficient for use in a real-time application and efficiently tracked differences in animal activity. In their study, the developed CVS was able to track the distance traveled and the walking behavior (path) identifying differences between a pig that received apomorphine or not. Similar approaches based on traditional imaging thresholding and frame by frame comparisons were also used with broilers for the identification of flock behavior over time (82) and at different feeders (83).

Animal tracking and activity-related traits are still of great interest, with the identification and tracking of multiple animals and their interactions as one of the biggest issues. In order 
to overcome this challenge, a successful approach in pigs was to identify the animals by ellipsis fitting on the animal area and recognition of patterns printed on their backs (88). By using this simple approach, researchers were able to track and identify multiple animals with an accuracy of $88.7 \%$, enabling the characterization and evaluation of simple activity status as active or non-active with high correlation (mean of 0.9 ) with evaluations made by a human observer $(88,100)$. However, this identification and tracking approach cannot be used for animals with darker skin or in commercial farms that have animals with different skin colors since the method was developed for white pigs on a dark floor background and using a surveillance camera. Another challenge in the use of patterns printed on the animal's skin for identification in commercial settings is the higher stocking density and pen size. In an attempt to solve the issue of multiple animal tracking, Matthews et al. (89) developed an approach using multiple 3D cameras to track multiple pigs in a pen and record their behavior, achieving an overall tracking accuracy of 0.89 . In a more recent study, a deep learning approach has been tested for identification and tracking of multiple pigs using standard digital cameras, achieving a precision of 0.91 and recall rate of 0.67 on a test data set of pigs under challenging floor and lightening conditions (90). Even though these are promising results, these systems are prone to lose animal tracking over time, without a current solution on how to get back to the correct tracking of each animal. Thus, there is still the need for a reliable CVS capable of identification and tracking of individual animals in farm conditions.

In order to identify more behaviors, like feeding and drinking, manual segmentation of the captured image in regions of interest (ROI) have proved effective (91). The basic concept is to identify not only the animals but also objects, such as the water source and feeders, and track how animals interact with those objects. Using this technique associated with a transfer function model, with a single input and single output, Kashiha et al. (91) were able to identify pig drinking behavior with an $R^{2}$ of 0.92 on a single dataset with 40 pigs divided in 4 pens. Machine learning techniques have proven efficient for the identification of animal posture such as standing, lying, or sitting $(85,93)$. In their study, Barnard et al. (85) achieved a mean accuracy of 0.91 when using a structural support vector machine to classify dog postures from depth images. In another study, Lao et al. (93) defined a classification tree for identification of several sow behaviors using videos from 3D cameras with high (99\%) accuracy for lying, sitting, and drinking behaviors and lower for kneeling (78\%) and shifting (64\%). Machine learning techniques have also shown to be powerful for the identification of social interactions among animals, such as mounting and aggressive behavior (92, 94). Viazzi et al. (92) achieved a mean accuracy of 0.88 when using linear discriminant analysis for classifying aggressive behavior in pigs, while Chen et al. (94) achieved an accuracy of 0.97 on the validation set using a convolution neural network and long shortterm memory approach. Even though there was an improvement in accuracy in the latter study, it did not include an automated strategy for the identification of individual animals. Thus, current methods can be used for the identification of behavior changes on group level, but not on individual level. Another aspect that must be highlighted here is that the methods discussed above are supervised learning approaches, and as such, they need a dataset of labeled images (ground truth) for the training step. In order to produce those training datasets, manual classification of the images by a human observer is needed, thus the model will be at most as good as the human observer who evaluated the images in the first place. One way to improve the gold standard used in such methods is by the development and adoption of well-defined methodologies for the measurement and record of traits of interest, followed by regular training and testing of the human evaluators to increase intra and inter-evaluator reliability. Another approach that can also be used is crowdsourcing the development of the dataset. With crowdsourcing, the manual classification of the images can be done by several evaluators and using majority vote, so reducing the impact of individual evaluators' subjectivity (101). Another approach that has shown improvement of the predictive accuracy is the use of multimodel prediction, also known as model assemble methods. One of the most basic assemble would be the use of the average predicted value from multiple models (102). The combination of crowdsourcing the dataset development with the use of multiple model classifiers has allowed an increase in accuracy for applications in medical image analysis, with an artificial intelligence system outperforming trained evaluators (103).

\section{Identification of Mastitis and Digital Dermatitis by Thermography}

So far, most of the computer vision applications presented have used standard CCD or CMOS cameras. As previously discussed in section Images on the Visible Light Spectrum, there are also other sensors of interest, such as thermal and depth cameras. Thermal imaging cameras are commonly used in veterinary sciences as a diagnostic tool in clinical examination. The images can be used to identify differences in external/skin temperature that can be related to inflammatory process, infection, necrosis, stress, and overall health. In research, infrared thermography (IRT) has been used to identify mastitis in dairy cattle and sheep $(63,64)$, and for digital dermatitis in sheep (68), showing the capability to classify healthy and clinically sick animals. Moreover, in a controlled study Metzner et al. (65) observed that IRT was capable of detecting an increase in udder temperature $\sim 10$ h after inoculation with $E$. coli. Nevertheless, these were clinical trial studies with a limited number of animals, thus there is still the need to evaluate IRT under more general farm conditions. Zaninelli et al. (66) evaluated the use of IRT for udder health using data from more than 300 cows from three farms. In their study, even though the images were collected manually, the imaging processing was automated using a classical image threshold for measurement of udder temperature. In this initial step toward automation of udder health evaluation, a threshold model was developed for the classification of udder health in two categories, achieving an area under the curve (AUC) around 0.8. In another study, 149 cows from eight farms were clinically evaluated for digital dermatitis, and IRT was evaluated as a non-invasive field diagnostic tool for dairy cattle (67). In their study, an AUC of 0.84 for the receiver operating characteristic (ROC) curve was observed for classification on the 
temperature difference from the front and rear feet, showing promises of IRT as an on-farm tool. However, the authors pointed out that images were collected manually and that in $11 \%$ of the cows' data had to be removed due to excessive dirt. Also, there are still many complications related to IRT measurement variation and repeatability. In some studies, it was observed that animal skin/surface temperature can vary according to external factors, such as environmental temperature, wind speed, or other factors, such as operator and camera positioning, and body region evaluated (69-71). Thus, IRT applications have been challenging, and they are still generally based on semi-automated CVS, so that additional effort should be placed on developing methods for automation and improvement of measurements for on farm conditions.

\section{Evaluation of Animal Surface and Related Traits Using 3D Cameras}

The interest in the use of $3 \mathrm{D}$ cameras is due to the capability of measuring traits in the 3-dimensional space such as animal, body position, gait, and volume. Also, for some applications, there is an improvement of image processing since, within $3 \mathrm{D}$ images, there is less noise due to light and background conditions and it is easier to use the distance to the camera as a threshold. Regarding the uses of depth sensors, there are many examples of successful applications including tracking of fish within a tank (79), identification of landmarks on animal shape with consecutive modeling of gait $(72,99)$, body condition score $(78)$, sickness detection (84), and the estimation of many other body measurements that will be discussed below.

Studies in gait analysis usually demanded intense manual labeling of video frames by a human observer and/or an expensive system of plate markers to be positioned on the animal body and multiple cameras (98). However, with the introduction of time of flight technology and more accessible 3D cameras, CVS with a single or two sensors were capable of efficiently estimate walking kinematics in pigs in a cost-effective framework with prediction accuracy comparable to the state of the art of kinematics systems $\left(R^{2}=0.99\right)(99)$.

Spoliansky et al. (78) developed an automated CVS based on $3 \mathrm{D}$ cameras for the evaluation of dairy cows' body condition score (BCS). In this study, top view images were collected from cows at the moment they were leaving the milking parlor. These images were then automatically processed with removal of background, rotation, and centralization of the cow, holes filled, and normalization. Several image features were extracted from the processed images and used for the development of multiple linear regression models via stepwise regression. Even though the variables extracted did not present a high correlation with BCS, the developed model achieved an average $R^{2}$ of 0.68 , which is comparable or better than previous studies based on manual processing of the images using either standard digital images $(75,76)$ or thermal cameras $(77)$.

In chickens, 3D sensors have also been used to identify small modifications in the animal surface that is related to head and tail positioning (84). In this study, in which animals were challenged with the Newcastle disease virus, it was possible to identify alterations in the animal shape and behavior 6 days after the inoculation.

Other applications in which depth sensors are showing promising results are for estimation of animal body measurements (heights, widths, area, and volume as examples) and body weight $(44,96,97)$. In one study, a CVS based on depth image could extract additional information on the animal volume, achieving an $R^{2}$ of 0.99 (96) under experimental conditions. In another study (97), depth cameras were evaluated for estimation of body measurements on pigs in farm conditions, achieving high $R^{2}(0.77-0.93)$ between the manual measurement and the measures estimated from the images. Nevertheless, these previous studies used some level of manual handling of the images and they did not evaluate model performance using an independent set of animals or a cross-validation approach. This hampers the evaluation of how generalizable the prediction models are, that is, how these CVS based on 3D cameras would perform in practice. Another drawback of these previous studies is the lack of automation for application in farm conditions, where it would be extremely difficult to manually process the images.

\section{Automated Prediction of Individual Body Measurements}

Automated non-contact prediction of body weight and body measurements is a long-desired application for many animal production systems. Kashiha et al. (95) developed a CVS for automated prediction of BW in pigs under experimental conditions achieving good prediction $\left(R^{2}=0.97\right)$ for body weight using surveillance cameras. However, as stated by the authors, this previous method was still restricted by background and light conditions, along with animal coat color. Recently, Fernandes et al. (14) developed an automated CVS based on depth camera for real-time video processing and prediction of body weight in live pigs. They worked with videos collected under farm conditions using multiple linear regression models with features extracted from the images as predictor variables, achieving high predictive accuracy evaluated with cross-validation $\left(R^{2}=0.92\right)$. An adaptation of their CVS was also evaluated for prediction of body weight in beef cattle from depth images (73) achieving high $R^{2}(0.79-0.91)$ with an artificial neural network approach. In both works, the images were collected from animals partially restrained and there was only one animal in the camera field of view so that future developments with the CVS on barn conditions would be necessary for better evaluation.

There are also many attempts to develop CVS for automated prediction of body weight and body measurements in fish, where the main challenges are related to fish body positioning and segmentation, and external factors such as light and background conditions. To tackle these issues, one study in halibut developed a CVS based on multi-scale body contour matching and completion using a double local threshold model with body shape priors (80). The final model developed was able to estimate the fish body with an average intersection over union (IoU) of 95.6\%. In another study in Nile tilapia, Fernandes et al. (81) used a deep learning approach for fish body segmentation from images under different lighting and background conditions. 
The approach was able to distinguish fish from the background with a validation IoU of $99 \%$, and for the fish body from fins and background of $0.91 \%$. In their study, the final fish body area was then used for prediction of fish body weight, achieving a predictive $R^{2}$ of 0.96 . Nevertheless, in this study, the fish were removed from the water, while in the previous one images from the top view of fish in a shallow water area were used. Hence, it is still necessary to develop CVSs that can evaluate fish underwater inside production cages. This adds more challenges, such as interference and occlusion due to different water transparency.

\section{Perspectives of CVS for High-Throughput Phenotyping}

CVS together with other sensor technologies are at the forefront of precision livestock farming, with some systems already been implemented in farm applications (104). Such systems have the potential to enable high-throughput phenotyping (HTP), which can be defined as the measurement of a single or many different traits of interest at multiple times during the animal life. HTP applications promise the generation of large amounts of data that will improve the accuracy of current methods and open a myriad of opportunities to advance breeding programs and livestock production (105). Nevertheless, for their implementation on breeding programs, there is the need to develop automated and robust CVS that are capable of collecting, processing, analyzing, and transmitting individual animal data. For this to happen, several key resources and tools must be developed such as improvement of rural broadbands, data integration, data mining, and novel predictive tools among others (106). A specific strategy used to circumvent the issue of individual animal identification is by using other technologies such as RFID tags, associated with the CVS (72). Nevertheless, there is still room for more progress in the use of image analysis for animal identification. Recent developments in machine learning algorithms for image analysis such as deep learning have shown promising results in other areas such as human face recognition, disease detection, and classification, among others (22). Generally, these algorithms demand very large datasets to be trained such as the Microsoft Common Objects in Context (COCO) (107). Nonetheless, with techniques such as transfer learning of pretrained models, we expect that deep learning may play an important role in the future development of CVS applications for animal production.

Animal phenotyping, or rather, the measurement of traits of interest, has long been a constant and important practice in animal management and also for the development of breeding programs for different animal production systems. In this manuscript, we discussed CVS as an interesting tool for the collection of such phenotypes without direct interaction with the animals. Thus, in the last decade, several efforts have been made toward the measurement of group-level traits, such as group growth, activity, drinking and feeding behavior, and animal spatial distribution among others with most of the successful applications based on standard digital cameras implementing classic image analysis and machine learning algorithms. Nevertheless, most of the works in the literature deals with a small group of animals, with just a few works evaluating CVS in farm environments $(14,66,67,97)$ or under challenging light and background conditions $(80,81,90)$ with the application of more sophisticated machine learning algorithms.

Nevertheless, there are already some examples of how CVS can be leveraged by breeding programs. In a study by Moore et al. (108), data from 17,765 image carcass records of prime cuts and carcass weight of commercial beef slaughter was used to predict genetic parameters in beef cattle. The authors concluded that by leveraging the information from the CVS it was possible to yield more accurate genetic parameters due to the higher volume of data. In another study, Nye et al. (74) developed a web scraper and an image segmentation algorithm to extract images and information from breeding programs catalogs. The information retrieved was used in a subsequent step to predict genetic parameters related to coat pigmentation and conformation traits in dairy cattle. The authors demonstrated that, for dairy cattle, approximately only 50 images were required to train their semisupervised machine learning approach.

\section{CONCLUDING REMARKS}

The idea of developing CVSs for automatic monitoring and measuring traits of interest in animals is not new. Early developments in digital image analysis and computer vision have shown the potential of the use of images to evaluate animal behavior, gait, body weight, and other traits in experimental conditions, with some more recent studies evaluating also on-farm applications. Also, there are studies showing that different imaging technologies can be better suited for specific applications, such as IRT for identification of mastitis and digital dermatitis in dairy cattle, or spectral and hyperspectral imaging in food sciences. However, there is also a great number of attempts to develop CVS based on more accessible technologies such as standard digital cameras and 3D cameras.

Applications of CVS in animal and veterinary sciences are currently a growing research area. Even though there are already some commercial products for monitoring groups of live animals, or slaughtered animals at the abattoir, there are still several challenges that demand intense research for the successful development and deployment of practical solutions. Current challenges involve the development and implementation of reliable CVS for the autonomous acquisition of data regarding single or multiple traits in farm conditions, as there are still few studies that evaluated these CVS using validation data sets, including different animals in the same farm or across multiple farms. Another area of importance is individual animal identification and tracking since most of the currently developed methods are still prone to error. There is also the need for the development of methods to connect the increasing number of devices used for different applications. This may enable the implementation of more sophisticated predictive algorithms based on multiple inputs and multiple outputs (joint prediction of multiple traits). Finally, there is the need for the development of applications for the delivery of the information generated 
by the CVS to connected systems thus generating valuable information to farmers and managers. This is the focus of areas such as big data and internet of things which, even though are not the focus of this review, these areas are going to be indispensable for the further development of CVS animal breeding programs and production systems.

\section{AUTHOR CONTRIBUTIONS}

AF, JD, and GR contributed to the conception and design of the review. AF wrote the first draft of the manuscript. All authors

\section{REFERENCES}

1. Gregory RL. Eye and Brain: The psychology of seeing. Third. New York: McGraw-Hill (1978). Available online at: https://sofamphoto.files.wordpress. com/2014/09/eye-brain-gregory.pdf (accessed August 24, 2017).

2. Burger W, Burge MJ. Digital image processing: An Algorithmic Introduction Using Java. 2nd ed. London: Springer London (2005). p. 811.

3. Atherton TJ, Kerbyson DJ. Size invariant circle detection. Image Vis Comput. (1999) 17:795-803. doi: 10.1016/S0262-8856(98)00160-7

4. Kheradmand A, Milanfar P. Non-linear structure-aware image sharpening with difference of smoothing operators. Front ICT. (2015) 2:22. doi: $10.3389 /$ fict.2015.00022

5. Rosin PL, Ioannidis E. Evaluation of global image thresholding for change detection. Pattern Recognit Lett. (2003) 24:2345-56. doi: 10.1016/S0167-8655(03)00060-6

6. Polesel A, Ramponi G, Mathews VJ. Image enhancement via adaptive unsharp masking. IEEE Trans Image Process. (2000) 9:505-10. doi: $10.1109 / 83.826787$

7. Nakagomi K, Shimizu A, Kobatake H, Yakami M, Fujimoto K, Togashi K. Multi-shape graph cuts with neighbor prior constraints and its application to lung segmentation from a chest CT volume. Med Image Anal. (2013) 17:62-77. doi: 10.1016/j.media.2012.08.002

8. Tang M, Gorelick L, Veksler O, Boykov Y. GrabCut in one cut. In: Proceedings of "International Conference on Computer Vision" (ICCV) (Sydney), 8. (2013). Available online at: http://www.csd.uwo.ca//simyuri/ Papers/iccv13_one_cut.pdf (accessed March 8, 2017).

9. Szelisk R. Computer vision : algorithms applications. In: Gries D, Schneider FB. London: Springer-Verlag (2011). doi: 10.1007/978-1-84882-935-0

10. Hough PVC. Method and Means For Recognizing Complex Patterns (1962). Available online at: https://www.osti.gov/scitech/biblio/4746348 (accessed April 17, 2017).

11. Bay H, Tuytelaars T, Van Gool L. SURF: Speeded Up Robust Features. In: European Conference on Computer Vision (Berlin, Heidelberg: Springer) (2006). p. 404-417. doi: 10.1007/11744023_32

12. Leutenegger S, Chli M, Siegwart RY. BRISK: binary robust invariant scalable keypoints. In: International Conference on Computer Vision (Barcelona: IEEE). (2011). p. 2548-55. doi: 10.1109/ICCV.2011.6126542

13. Murphy KP. Machine Learning: A Probabilistic Perspective. Cambridge, Massachusetts: MIT Press (2012). Available at: http://mitpress.mit.edu (accessed June 18, 2019).

14. Fernandes AFA, Dórea JRR, Fitzgerald R, Herring W, Rosa GJM. A novel automated system to acquire biometric and morphological measurements and predict body weight of pigs via 3D computer vision. J Anim Sci. (2019) 97:496-508. doi: 10.1093/jas/sky418

15. Boyle WS, Smith GE. Buried channel charge coupled devices. 9. (1973). Available online at: https://patents.google.com/patent/US3792322 (accessed May 17, 2019]

16. Lister M. (1995). The photographic image in digital culture. London: Routledge

17. van Dijck J. Digital photography: communication, identity, memory. Vis Commun. (2008) 7:57-76. doi: 10.1177/1470357207084865 contributed to manuscript revision, read, and approved the submitted version.

\section{FUNDING}

The authors thank the financial support from the Coordination for the Improvement of High Education Personnel (CAPES), Brazil ordinance (49/2013), the Wisconsin Agriculture Experiment Station Hatch grant (142-AAC7939), and United States Department of Agriculture (USDA).
18. Viola P, Jones MJ. Robust Real-Time Face Detection. Int J Comput Vis. (2004) 57:137-54. doi: 10.1023/B:VISI.0000013087.49260.fb

19. LeCun Y, Bottou L, Bengio Y, Haffner P. Gradient-based learning applied to document recognition. Proc IEEE. (1998) 86:2278-324. doi: 10.1109/5.726791

20. Litjens G, Sánchez CI, Timofeeva N, Hermsen M, Nagtegaal I, Kovacs I, et al. Deep learning as a tool for increased accuracy and efficiency of histopathological diagnosis. Sci Rep. (2016) 6:26286. doi: 10.1038/srep26286

21. Goodfellow I, Bengio Y, Courville A. Deep Learning. Cambridge, MS: MIT Press (2016).

22. LeCun Y, Bengio Y, Hinton G. Deep learning. Nature. (2015) 521:436-44. doi: $10.1038 /$ nature 14539

23. Hyndman RJ, Koehler AB. Another look at measures of forecast accuracy. Int J Forecast. (2006) 22:679-88. doi: 10.1016/j.ijforecast.2006.03.001

24. Aggarwal CC. (2015). Data Mining: The Textbook. New York, NY: Springer. doi: 10.1007/978-3-319-14142-8

25. Hastie T, Tibshirani R, Friedman J. The Elements of Statistical Learning. Second Edition. New York, NY: Springer New York (2009). doi: 10.1007/978-0-387-84858-7

26. Helmers $H$, Schellenberg M. CMOS vs. CCD sensors in speckle interferometry. Opt Laser Technol. (2003) 35:587-95. doi: 10.1016/S0030-3992(03)00078-1

27. Ring F, Jung A, Zuber J. Infrared Imaging A Casebook in Clinical Medicine. Bristol-UK: IOP Publishing. (2015). doi: 10.1088/978-0-7503-1143-4

28. Lavers C, Franks K, Floyd M, Plowman A. Application of remote thermal imaging and night vision technology to improve endangered wildlife resource management with minimal animal distress and hazard to humans. J Phys Conf Ser. (2005) 15:207-12. doi: 10.1088/1742-6596/15/1/035

29. Swanson GA, Sargeant AB. Observation of nighttime feeding behavior of ducks. J Wildl Manage. (1972) 36:959-61. doi: 10.2307/3799457

30. McManus C, Tanure CB, Peripolli V, Seixas L, Fischer V, Gabbi AM, et al. Infrared thermography in animal production: an overview. Comput Electron Agric. (2016) 123:10-6. doi: 10.1016/j.compag.2016.01.027

31. Zanuttigh P, Mutto CD, Minto L, Marin G, Dominio F, Cortelazzo GM. Time-of-Flight and Structured Light Depth Cameras: Technology and Applications. Cham: Springer International Publishing (2016). p. 355. doi: 10.1007/978-3-319-30973-6

32. Giancola S, Valenti M, Sala R. A survey on 3D cameras: metrological comparison of time-of-flight, structured-light and active stereoscopy technologies. In: SpringerBriefs in Computer Science (Cham: Springer) (2018). p. 90. doi: 10.1007/978-3-319-91761-0

33. Yoo HW, Druml N, Brunner D, Schwarzl C, Thurner T, Hennecke M, et al. MEMS-based lidar for autonomous driving. Elektrot Informat. (2018) 135:408-15. doi: 10.1007/s00502-018-0635-2

34. Xiong Z, Sun D-W, Zeng X-A, Xie A. Recent developments of hyperspectral imaging systems and their applications in detecting quality attributes of red meats: a review. J Food Eng. (2014) 132:1-13. doi: 10.1016/j.jfoodeng.2014.02.004

35. Tao F, Ngadi M. Recent advances in rapid and nondestructive determination of fat content and fatty acids composition of muscle foods. Crit Rev Food Sci Nutr. (2018) 58:1565-93. doi: 10.1080/10408398.2016.1261332 
36. Ozaki Y, McClure WF, Christy AA (editors). Near-Infrared Spectroscopy in Food Science and Technology. Hoboken, NJ: JohnWiley \& Sons, Inc. (2007). p. 11-46. doi: 10.1002/0470047704

37. Font-i-Furnols M, Carabús A, Pomar C, Gispert M. Estimation of carcass composition and cut composition from computed tomography images of live growing pigs of different genotypes. Animal. (2015) 9:166-78. doi: $10.1017 /$ S1751731114002237

38. Scholz AM, Bünger L, Kongsro J, Baulain U, Mitchell AD. Noninvasive methods for the determination of body and carcass composition in livestock: dual-energy X-ray absorptiometry, computed tomography, magnetic resonance imaging and ultrasound: invited review. Animal. (2015) 9:1250-64. doi: 10.1017/S1751731115000336

39. Lucas D, Brun A, Gispert M, Carabús A, Soler J, Tibau J, et al. Relationship between pig carcass characteristics measured in live pigs or carcasses with Piglog, Fat-o-Meat'er and computed tomography. Livest Sci. (2017) 197:8895. doi: 10.1016/J.LIVSCI.2017.01.010

40. Lin SSP. Automated Pattern Recognition of Beef. (1978). Available online at: http://krex.k-state.edu/dspace/bitstream/handle/2097/13198/ LD2668R41978L54.pdf? sequence=1 (accessed August 29, 2017).

41. Cross HR, Gilliland DA, Durland PR, Seideman S. Beef carcass evaluation by use of a video image analysis system. J Anim Sci. (1983) 57:908-17. doi: $10.2527 /$ jas1983.574908x

42. Wassenberg RL, Allen DM, Kemp KE. Video image analysis prediction of total kilograms and percent primal lean and fat yield of beef carcasses. J Anim Sci. (1986) 62:1609-16. doi: 10.2527/jas1986.6261609x

43. Teixeira A, Joy M, Delfa R. In vivo estimation of goat carcass composition and body fat partition by real-time ultrasonography. J Anim Sci. (2008) 86:2369-76. doi: 10.2527/jas.2007-0367

44. Gomes RA, Monteiro GR, Assis GJF, Busato KC, Ladeira MM, Chizzotti ML. Technical note: estimating body weight and body composition of beef cattle trough digital image analysis. J Anim Sci. (2016) 94:5414-22. doi: 10.2527/jas.2016-0797

45. Nunes J, Piquerez M, Pujadas L, Armstrong E, Fernández A, Lecumberry F. Beef quality parameters estimation using ultrasound and color images. BMC Bioinformatics. (2015) 16 (Suppl. 4):S6. doi: 10.1186/1471-2105-16-S4-S6

46. Jackman P, Sun D-W, Allen P, Brandon K, White A-M. Correlation of consumer assessment of longissimus dorsi beef palatability with image colour, marbling and surface texture features. Meat Sci. (2010) 84:564568. doi: 10.1016/j.meatsci.2009.10.013

47. Folkestad A, Wold JP, Rørvik, K.-A., Tschudi J, Haugholt KH, Kolstad K, et al. Rapid and non-invasive measurements of fat and pigment concentrations in live and slaughtered Atlantic salmon (Salmo salar L.). Aquaculture. (2008) 280:129-35. doi: 10.1016/j.aquaculture.2008.04.037

48. Paluchowski LA, Misimi E, Grimsmo L, Randeberg LL. Towards automated sorting of Atlantic cod (Gadus morhua) roe, milt, and liver - Spectral characterization and classification using visible and near-infrared hyperspectral imaging. Food Control. (2016) 62:337-45. doi: 10.1016/j.foodcont.2015.11.004

49. Sture $\varnothing, \emptyset$ ye ER, Skavhaug A, Mathiassen JR. A 3D machine vision system for quality grading of Atlantic salmon. Comput Electron Agric. (2016) 123:142-8. doi: 10.1016/j.compag.2016.02.020

50. Khoshnoudi-Nia S, Moosavi-Nasab M. Prediction of various freshness indicators in fish fillets by one multispectral imaging system. Sci Rep. (2019) 9:14704. doi: 10.1038/s41598-019-51264-z

51. Zapotoczny P, Szczypiński PM, Daszkiewicz T. Evaluation of the quality of cold meats by computer-assisted image analysis. LWT - Food Sci Technol. (2016) 67:37-49. doi: 10.1016/j.lwt.2015.11.042

52. Adamczak L, Chmiel M, Florowski T, Pietrzak D, Witkowski M, Barczak $\mathrm{T}$. The use of $3 \mathrm{D}$ scanning to determine the weight of the chicken breast. Comput Electron Agric. (2018) 155:394-9. doi: 10.1016/j.compag.2018. 10.039

53. Guanjun B, Mimi J, Yi X, Shibo C, Qinghua Y. Cracked egg recognition based on machine vision. Comput Electron Agric. (2019) 158:159-66. doi: 10.1016/j.compag.2019.01.005

54. Doeschl-Wilson AB, Green DM, Fisher AV, Carroll SM, Schofield $\mathrm{CP}$, Whittemore CT. The relationship between body dimensions of living pigs and their carcass composition. Meat Sci. (2005) 70:229-40. doi: 10.1016/j.meatsci.2005.01.010
55. Alsahaf A, Azzopardi G, Ducro B, Hanenberg E, Veerkamp RF, Petkov N. Estimation of muscle scores of live pigs using a kinect camera. IEEE Access. (2019) 7:52238-45. doi: 10.1109/ACCESS.2019.29 10986

56. Fernandes AFA, Dórea JRR, Valente BD, Fitzgerald R, Herring W, Rosa GJM. Comparison of data analytics strategies in computer vision systems to predict pig body composition traits from 3D images. J Anim Sci. (2020) 98:1-9. doi: 10.1093/jas/skaa250

57. Wang X, Zhao M, Ju R, Song Q, Hua D, Wang C, et al. Visualizing quantitatively the freshness of intact fresh pork using acousto-optical tunable filter-based visible/near-infrared spectral imagery. Comput Electron Agric. (2013) 99:41-53. doi: 10.1016/j.compag.2013.08.025

58. Ostermeier GC, Sargeant GA, Yandell BS, Parrish JJ. measurement of bovine sperm nuclear shape using fourier harmonic amplitudes. J Androl. (2001) 22:584-94. doi: 10.1002/J.1939-4640.2001.TB02218.X

59. Sassi N, Ben, Averós X, Estevez I. Technology and poultry welfare. Animals. (2016) 6:62-83. doi: 10.3390/ani6100062

60. Li N, Ren Z, Li D, Zeng L. Review: automated techniques for monitoring the behaviour and welfare of broilers and laying hens: towards the goal of precision livestock farming. Animal. (2019) 14:617-25. doi: 10.1017/S1751731119002155

61. Nasirahmadi A, Edwards SA, Sturm B. Implementation of machine vision for detecting behaviour of cattle and pigs. Livest Sci. (2017) 202:25-38. doi: 10.1016/j.livsci.2017.05.014

62. Saberioon M, Gholizadeh A, Cisar P, Pautsina A, Urban J. Application of machine vision systems in aquaculture with emphasis on fish: state-of-the-art and key issues. Rev Aquac. (2017) 9:369-87. doi: 10.1111/raq.12143

63. Hovinen M, Siivonen J, Taponen S, Hänninen L, Pastell M, Aisla A-M, et al. Detection of clinical mastitis with the help of a thermal camera. J Dairy Sci. (2008) 91:4592-8. doi: 10.3168/jds.2008-1218

64. Martins RFS, do Prado Paim T, de Abreu Cardoso C, Stéfano Lima Dallago B, de Melo CB, Louvandini H, et al. Mastitis detection in sheep by infrared thermography. Res Vet Sci. (2013) 94:722-4. doi: 10.1016/j.rvsc.2012. 10.021

65. Metzner M, Sauter-Louis C, Seemueller A, Petzl W, Zerbe H. Infrared thermography of the udder after experimentally induced Escherichia coli mastitis in cows. Vet J. (2015) 204:360-2. doi: 10.1016/J.TVJL.2015.04.013

66. Zaninelli M, Redaelli V, Luzi F, Bronzo V, Mitchell M, Dell'Orto V, et al. First evaluation of infrared thermography as a tool for the monitoring of udder health status in farms of dairy cows. Sensors. (2018) 18:862. doi: $10.3390 / \mathrm{s} 18030862$

67. Alsaaod M, Syring C, Dietrich J, Doherr MG, Gujan T, Steiner A. A field trial of infrared thermography as a non-invasive diagnostic tool for early detection of digital dermatitis in dairy cows. Vet J. (2014) 199:281-5. doi: 10.1016/j.tvjl.2013.11.028

68. Byrne DT, Berry DP, Esmonde H, McGovern F, Creighton P, McHugh N. Infrared thermography as a tool to detect hoof lesions in sheep. Transl Anim Sci. (2019) 3:577-88. doi: 10.1093/tas/txy132

69. Byrne DT, Berry DP, Esmonde H, McHugh N. Temporal, spatial, inter-, and intra-cow repeatability of thermal imaging. J Anim Sci. (2017) 95:970-9. doi: $10.2527 /$ jas2016.1005

70. Yang C, Li G, Zhang X, Gu X. Udder skin surface temperature variation preand post-milking in dairy cows as determined by infrared thermography. $J$ Dairy Res. (2018) 85:201-3. doi: 10.1017/S0022029918000213

71. Scoley GE, Gordon AW, Morrison SJ. Use of thermal imaging in dairy calves: exploring the repeatability and accuracy of measures taken from different anatomical regions1. Transl Anim Sci. (2019) 3:564-76. doi: $10.1093 /$ tas/txy126

72. Salau J, Haas JH, Junge W, Thaller G. Automated calculation of udder depth and rear leg angle in Holstein-Friesian cows using a multi-Kinect cow scanning system. Biosyst Eng. (2017) 160:154-69. doi: 10.1016/j.biosystemseng.2017.06.006

73. Cominotte A, Fernandes AFA, Dorea JRR, Rosa GJM, Ladeira MM, van Cleef EHCB, et al. Automated computer vision system to predict body weight and average daily gain in beef cattle during growing and finishing phases. Livest Sci. (2020) 232:103904-14. doi: 10.1016/j.livsci.2019.103904

74. Nye J, Zingaretti LM, Pérez-Enciso M. Estimating conformational traits in dairy cattle with deepaps: a two-step deep learning automated 
phenotyping and segmentation approach. Front Genet. (2020) 11:513. doi: $10.3389 /$ fgene.2020.00513

75. Bewley JM, Peacock AM, Lewis O, Boyce RE, Roberts DJ, Coffey MP, et al. Potential for estimation of body condition scores in dairy cattle from digital images. J Dairy Sci. (2008) 91:3439-53. doi: 10.3168/jds.20 07-0836

76. Bercovich A, Edan Y, Alchanatis V, Moallem U, Parmet Y, Honig H, et al. Development of an automatic cow body condition scoring using body shape signature and Fourier descriptors. J Dairy Sci. (2013) 96:8047-59. doi: 10.3168/jds.2013-6568

77. Halachmi I, Klopči,č M, Polak P, Roberts DJ, Bewley JM. Automatic assessment of dairy cattle body condition score using thermal imaging. Comput Electron Agric. (2013) 99:35-40. doi: 10.1016/j.compag.2013.08.012

78. Spoliansky R, Edan Y, Parmet Y, Halachmi I. Development of automatic body condition scoring using a low-cost 3-dimensional Kinect camera. J Dairy Sci. (2016) 99:7714-25. doi: 10.3168/jds.2015-10607

79. Saberioon MM, Cisar P. Automated multiple fish tracking in threeDimension using a Structured Light Sensor. Comput Electron Agric. (2016) 121:215-21. doi: 10.1016/j.compag.2015.12.014

80. Wang G, Hwang J-N, Wallace F, Rose C. Multi-scale fish segmentation refinement and missing shape recovery. IEEE Access. (2019) 7:52836-45. doi: 10.1109/ACCESS.2019.2912612

81. Fernandes AFA, Turra EM, de Alvarenga ÉR, Passafaro TL, Lopes FB, Alves GFO, et al. Deep Learning image segmentation for extraction of fish body measurements and prediction of body weight and carcass traits in Nile tilapia. Comput Electron Agric. (2020) 170:105274. doi: 10.1016/j.compag.2020.105274

82. Kashiha M, Pluk A, Bahr C, Vranken E, Berckmans D. Development of an early warning system forabroiler house using computer vision. Biosyst Eng. (2013) 116:36-45. doi: 10.1016/j.biosystemseng.2013.06.004

83. Neves DP, Mehdizadeh SA, Tscharke M, Nääs I, de A, Banhazi TM. Detection of flock movement and behaviour of broiler chickens at different feeders using image analysis. Inf Process Agric. (2015) 2:177-82. doi: 10.1016/j.inpa.2015.08.002

84. Okinda C, Lu M, Liu L, Nyalala I, Muneri C, Wang J, et al. A machine vision system for early detection and prediction of sick birds: a broiler chicken model. Biosyst Eng. (2019) 188:229-42. doi: 10.1016/j.biosystemseng.2019.09.015

85. Barnard S, Calderara S, Pistocchi S, Cucchiara R, Podaliri-Vulpiani M, Messori S, et al. Quick, accurate, smart: 3d computer vision technology helps assessing confined animals' behaviour. PLoS ONE. (2016) 11:e0158748. doi: 10.1371/journal.pone. 0158748

86. Tillett RD, Onyango CM, Marchant JA. Using model-based image processing to track animal movements. Comput Electron Agric. (1997) 17:249-61. doi: 10.1016/S0168-1699(96)01308-7

87. Lind NM, Vinther M, Hemmingsen RP, Hansen AK. Validation of a digital video tracking system for recording pig locomotor behaviour. J Neurosci Methods. (2005) 143:123-32. doi: 10.1016/j.jneumeth.2004.09.019

88. Kashiha MA, Bahr C, Ott S, Moons CPH, Niewold TA, Ödberg FO, et al. Automatic identification of marked pigs in a pen using image pattern recognition. Comput Electron Agric. (2013) 93:111-20. doi: 10.1016/j.compag.2013.01.013

89. Matthews SG, Miller AL, PlÖtz T, Kyriazakis I. Automated tracking to measure behavioural changes in pigs for health and welfare monitoring. $S c i$ Rep. (2017) 7:17582. doi: 10.1038/s41598-017-17451-6

90. Psota ET, Mittek M, Pérez LC, Schmidt T, Mote B. Multi-pig part detection and association with a fully-convolutional network. Sensors. (2019) 19:852. doi: 10.3390/s19040852

91. Kashiha MA, Bahr C, Haredasht SA, Ott S, Moons CPH, Niewold TA, et al. The automatic monitoring of pigs water use by cameras. Comput Electron Agric. (2013) 90:164-9. doi: 10.1016/j.compag.2012.09.015

92. Viazzi S, Ismayilova G, Oczak M, Sonoda LT, Fels M, Guarino M, et al. Image feature extraction for classification of aggressive interactions among pigs. Comput Electron Agric. (2014) 104:57-62. doi: 10.1016/j.compag.2014.03.010

93. Lao F, Brown-Brandl T, Stinn JP, Liu K, Teng G, Xin H. Automatic recognition of lactating sow behaviors through depth image processing.
Comput Electron Agric. (2016) 125:56-62. doi: 10.1016/j.compag.2016. 04.026

94. Chen C, Zhu W, Steibel J, Siegford J, Wurtz K, Han J, et al. Recognition of aggressive episodes of pigs based on convolutional neural network and long short-term memory. Comput Electron Agric. (2020) 169:105166. doi: 10.1016/j.compag.2019.105166

95. Kashiha MA, Bahr C, Ott S, Moons CPH, Niewold TA, Ödberg FO, et al. Automatic weight estimation of individual pigs using image analysis. Comput Electron Agric. (2014) 107:38-44. doi: 10.1016/j.compag.2014.06.003

96. Kongsro J. Estimation of pig weight using a Microsoft Kinect prototype imaging system. Comput Electron Agric. (2014) 109:32-5. doi: 10.1016/j.compag.2014.08.008

97. Pezzuolo A, Guarino M, Sartori L, González LA, Marinello F. Onbarn pig weight estimation based on body measurements by a Kinect v1 depth camera. Comput Electron Agric. (2018) 148:29-36. doi: 10.1016/J.COMPAG.2018.03.003

98. Stavrakakis S, Guy JH, Warlow OME, Johnson GR, Edwards SA. Walking kinematics of growing pigs associated with differences in musculoskeletal conformation, subjective gait score and osteochondrosis. Livest Sci. (2014) 165:104-13. doi: 10.1016/j.livsci.2014.04.008

99. Stavrakakis S, Li W, Guy JH, Morgan G, Ushaw G, Johnson GR, et al. Validity of the Microsoft Kinect sensor for assessment of normal walking patterns in pigs. Comput Electron Agric. (2015) 117:1-7. doi: 10.1016/j.compag.2015.07.003

100. Ott S, Moons CPH, Kashiha MA, Bahr C, Tuyttens FAM, Berckmans D, et al. Automated video analysis of pig activity at pen level highly correlates to human observations of behavioural activities. Livest Sci. (2014) 160:132-7. doi: 10.1016/j.livsci.2013.12.011

101. Arganda-Carreras I, Turaga SC, Berger DR, Cireşan D, Giusti A, Gambardella LM, et al. Crowdsourcing the creation of image segmentation algorithms for connectomics. Front Neuroanat. (2015) 9:142. doi: 10.3389/fnana.2015.00142

102. Burnham KP, Anderson DR (editors). Model Selection and Multimodel Inference - A Pratical Information - Theoretic Approach. New York, NY: Springer New York (2004). p. 149-203. doi: 10.1007/b97636

103. McKinney SM, Sieniek M, Godbole V, Godwin J, Antropova N, Ashrafian H, et al. International evaluation of an AI system for breast cancer screening. Nature. (2020) 577:89-94. doi: 10.1038/s41586-019-1799-6

104. Berckmans D. General introduction to precision livestock farming. Anim Front. (2017) 7:6. doi: 10.2527/af.2017.0102

105. Rosa GJM, Dorea JRR, Fernandes AFA, Passafaro TL. 227 Leveraging on high-throughput phenotyping technologies to optimize livestock genetic improvement and husbandry. J Anim Sci. (2019) 97:55. doi: 10.1093/jas/skz258.111

106. Koltes JE, Cole JB, Clemmens R, Dilger RN, Kramer LM, Lunney $\mathrm{JK}$, et al. A vision for development and utilization of high-throughput phenotyping and big data analytics in livestock. Front Genet. (2019) 10:1197. doi: 10.3389/fgene.2019.01197

107. Lin, T.-Y., Maire M, Belongie S, Bourdev L, Girshick R, Hays J, et al. (2015). Microsoft COCO: Common Objects in Context. arXiv. Available online at: https://arxiv.org/abs/1405.0312 (accessed December 5, 2018).

108. Moore KL, Mrode R, Coffey MP. Genetic parameters of Visual Image Analysis primal cut carcass traits of commercial prime beef slaughter animals. Animal. (2017) 11:1653-9. doi: 10.1017/S1751731117000489

Conflict of Interest: The authors declare that the research was conducted in the absence of any commercial or financial relationships that could be construed as a potential conflict of interest.

Copyright (c) 2020 Fernandes, Dórea and Rosa. This is an open-access article distributed under the terms of the Creative Commons Attribution License (CC BY). The use, distribution or reproduction in other forums is permitted, provided the original author(s) and the copyright owner(s) are credited and that the original publication in this journal is cited, in accordance with accepted academic practice. No use, distribution or reproduction is permitted which does not comply with these terms. 